\title{
Timing and Carrier Synchronization With Channel Estimation in Multi-Relay Cooperative Networks
}

\author{
Ali A. Nasir, Student Member, IEEE, Hani Mehrpouyan, Member, IEEE, Steven D. Blostein, Senior Member, IEEE, \\ Salman Durrani, Senior Member, IEEE, and Rodney A. Kennedy, Fellow, IEEE
}

\begin{abstract}
Multiple distributed nodes in cooperative networks generally are subject to multiple carrier frequency offsets (MCFOs) and multiple timing offsets (MTOs), which result in time varying channels and erroneous decoding. This paper seeks to develop estimation and detection algorithms that enable cooperative communications for both decode-and-forward (DF) and amplify-and-forward (AF) relaying networks in the presence of MCFOs, MTOs, and unknown channel gains. A novel transceiver structure at the relays for achieving synchronization in AF-relaying networks is proposed. New exact closed-form expressions for the Cramér-Rao lower bounds (CRLBs) for the multi-parameter estimation problem are derived. Next, two iterative algorithms based on the expectation conditional maximization (ECM) and space-alternating generalized expectation-maximization (SAGE) algorithms are proposed for jointly estimating MCFOs, MTOs, and channel gains at the destination. Though the global convergence of the proposed ECM and SAGE estimators cannot be shown analytically, numerical simulations indicate that through appropriate initialization the proposed algorithms can estimate channel and synchronization impairments in a few iterations. Finally, a maximum likelihood (ML) decoder is devised for decoding the received signal at the destination in the presence of MCFOs and MTOs. Simulation results show that through the application of the proposed estimation and decoding methods, cooperative systems result in significant performance gains even in presence of impairments.
\end{abstract}

Index Terms-Cooperative communications, Cramér-Rao lower bounds (CRLB), expectation conditional maximization (ECM), space-alternating generalized expectation-maximization (SAGE), timing and carrier synchronization.

\section{INTRODUCTION}

C OOPERATIVE communications is an attractive low-cost solution to combat fading in wireless communications, where multiple single antenna relay terminals receive and cooperatively transmit the source information to the destination. In ideal settings, it has been shown that the same spatial cooperative diversity as that of multiple-input-multiple-output (MIMO)

Manuscript received May 25, 2011; revised August 31, 2011 and October 19, 2011; accepted October 22, 2011. Date of publication November 04, 2011; date of current version January 13, 2012. The associated editor for this manuscript was Prof. Mérouane Debbah. This work was supported by an ANU VC HDR Travel Grant, ANU International Ph.D. Scholarship, NSERC Discovery Grant 41731, and Vinnova Research and Innovation Fund for Sustainable Growth.

A. A. Nasir, S. Durrani, and R. Kennedy are with the Research School of Engineering, The Australian National University, Australia (e-mail: ali.nasir@anu. edu.au; salman.durrani@anu.edu.au; rodney.kennedy@anu.edu.au).

H. Mehrpouyan is with the Department of Signals and Systems, Chalmers University of Technology, Sweden (e-mail: hani.mehr@ieee.org).

S. D. Blostein is with the Department of Electrical and Computer Engineering, Queen's University, Canada (e-mail: steven.blostein@queensu.ca).

Digital Object Identifier 10.1109/TSP.2011.2174792 systems can be achieved in cooperative networks without the need for multiple antennas at each node [1]-[5]. In conventional MIMO systems, the antenna elements are collocated on a single device, which results in a single timing and carrier offset. However, in cooperative networks, multiple distributed nodes, each with its own local oscillator, gives rise to multiple carrier frequency offsets (MCFOs) and multiple timing offsets (MTOs) [6]-[8].

Most of the existing work in the literature focuses on estimating either MCFOs while assuming perfect timing synchronization [8]-[13] or MTOs while assuming perfect carrier synchronization [6], [7], [14], [15]. Cramér-Rao lower bounds (CRLBs) and different techniques for estimating MCFOs in MIMO systems are addressed in [9]-[12]. However, the algorithms in [9]-[12] are not applicable to the case of amplify-and-forward (AF)-relaying cooperative networks and are based on the assumption of perfect timing synchronization. Novel CRLBs and maximum likelihood (ML) estimators to obtain MTOs for detect-and-forward, decode-and-forward (DF), and AF systems are derived in [14], [15], and [6], [7], respectively. Similarly, in [8], [13], [16] the estimation of multiple channel gains and MCFOs in DF and AF cooperative systems are analyzed. However, the analyses in [6]-[16] are focused on estimating one set of system parameters while assuming that the remaining parameters are perfectly estimated and compensated. Admittedly, such assumptions do not hold in actual cooperative communication systems, where the channel gains, MCFOs, and MTOs need to be jointly estimated at the destination terminal.

Recently, a limited number of papers have investigated joint estimation of impairments. In [17], a new joint ML estimator for determining MCFOs, MTOs, and channel gains in DF cooperative networks is devised. Nevertheless, the ML estimator in [17] requires exhaustive search and is computationally very complex. In order to reduce the complexity of ML estimation, in [18] new iterative estimation schemes are proposed. However, the outcomes in [17], [18] are limited to DF cooperative networks, provide no specific initialization guidelines for the proposed estimators, and do not propose any means of decoding the received signals from multiple relays at the destination. Novel joint channel estimation and time-frequency synchronization for uplink orthogonal frequency-division multipleaccess (OFDMA) systems are proposed in [19]-[22] that exploit the cyclic prefix. However, depending on the number of subcarriers used, the frequency acquisition range of the algorithms in [19]-[22] is very limited.

Estimating MCFOs, MTOs, and unknown channel gains is the first step to achieving synchronization. In fact, in order to achieve synchronous and coherent communication in multi-relay 
cooperative networks, the estimated MCFOs, MTOs, and unknown channel gains need to be applied to successfully equalize the received signal at the destination node. Recent literature has addressed either MCFO compensation [23]-[25] or MTO compensation [6], [15]. In [23], a new class of distributed linear convolutional space-time codes are proposed to compensate the effect of MCFOs. However, the application of the codes in [23] reduce overall cooperative network throughput since their effective rate is less than one. In [24], [25], various novel algorithms for compensating and detecting the received signal at the destination in the presence of MCFOs are proposed. Nevertheless, the algorithms in [24], [25] can only detect the received signal at the destination over a narrow range of $\mathrm{CFO}$ values, e.g., $\left[-10^{-6}, 10^{-6}\right]$ in $[25]$. More importantly, the analyses in [23]-[25] focus on compensating one set of parameters and cannot equalize the received signal in the presence of both MCFOs and MTOs. Under the assumption of perfect frequency synchronization, a novel resynchronization filter is proposed in [6] and [15] to compensate the effect of MTOs in DF and AF cooperative systems, respectively. However, as anticipated and confirmed in this paper, in the presence of MCFOs, the resynchronization filter in [6] and [15] cannot compensate the effect of MTOs and results in erroneous decoding at the destination. In [26], a novel algorithm for compensating MCFOs and MTOs in time-division multiple-access (TDMA) distributed MIMO systems is proposed that exploits spatial diversity at the multi-antenna receiver. However, the method in [26] cannot be applied to the case of multi-relay single-input-single-output (SISO) cooperative networks, where multiple single antenna relays communicate with a single antenna destination. To the best of the authors' knowledge, an estimation and equalization scheme for cooperative networks in the presence of MCFOs, MTOs, and unknown channels has not been proposed in the existing literature.

In this paper, joint estimation of MCFOs, MTOs, and frequency flat-fading channel gains in both $\mathrm{DF}$ and $\mathrm{AF}$ multi-relay cooperative networks is addressed. A novel transceiver structure at the relays for achieving synchronization in AF-relaying networks is proposed and the signal model at the relays and destination in the presence of unknown channel gains, timing offsets, and frequency offsets is derived in detail. Next, the estimation problem is parameterized and the closed-form CRLBs for the multiple parameter estimation problem for both DF and AF cooperative networks are derived. A least squares (LS) estimator for joint estimation of MCFOs, MTOs, and channel gains in $\mathrm{AF}$ cooperative networks is proposed. In order to reduce the computational complexity associated with the estimation of synchronization parameters and channel gains, new iterative estimators based on expectation conditional maximization (ECM), and space-alternating generalized expectation-maximization (SAGE) are derived for both DF and AF cooperative networks. Finally, a new ML decoder for detecting the source signal at the destination in the presence of MCFOs and MTOs for both DF and AF cooperative systems is derived. Simulation results show that through the application of the proposed estimators and ML decoder, the performance gains promised by cooperative networks are reachable even in non-ideal settings. The contributions and organization of this paper can be summarized as follows.

- In Section II, a novel training and data transmission method for AF cooperative networks in the presence of

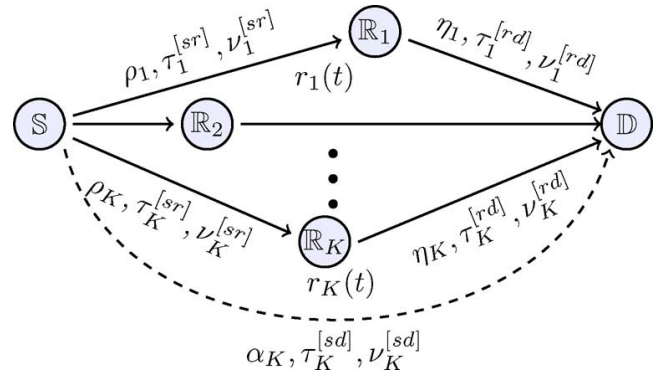

Fig. 1. The system model for the cooperative network.

MCFOs, MTOs, and multiple unknown channel gains is proposed.

- In Section III, new closed-form CRLBs for the multiple parameter estimation problem for both AF and DF cooperative networks are derived and used to assess the performance of the proposed estimators.

- In Section IV, an LS estimator for estimation of multiple system parameters in AF cooperative networks is derived. Simulation results show that the performance of the proposed LS algorithm and the ML estimator in [17] are close to the CRLB at mid-to-high SNR values. In order to achieve significantly reduced computational complexity, ECM and SAGE algorithms for estimation of multiple system parameters for both AF and DF cooperative systems are derived. Finally, the computational complexity of the proposed estimators is analyzed.

- In Section V, a new ML detector is derived that allows for the signal received from multiple relays to be successfully decoded in the presence of MCFOs and MTOs.

- In Section VI, numerical and simulation results are presented, where it is shown that the mean-square-error (MSE) performance of ML, and the proposed LS estimators for wide range of signal-to-noise-ratio (SNR) values are close to the derived CRLBs. Moreover, the MSE performance of the proposed ECM and SAGE estimators are evaluated. Finally, the effect of initialization on the performance of the proposed estimators is numerically investigated and specific guidelines that ensure the convergence of these estimators are outlined.

Notation: Superscripts $(\cdot)^{*},(\cdot)^{H}$ and $(\cdot)^{T}$ denote the conjugate, the conjugate transpose and the transpose operators, respectively. Bold face small letters, e.g., $\mathbf{x}$, are used for vectors, bold face capital alphabets, e.g., $\mathbf{X}$, are used for matrix representation. $\odot$ stands for Schur (element-wise) product, $|\cdot|$ is the modulus operator and $\|\mathbf{x}\|$ represents the $L_{2}$ norm of a vector $\mathbf{x} . \mathbb{E}\{\cdot\}$ denotes the expected value of its argument. $\Re\{\cdot\}$ and $\Im\{\cdot\}$ take the real and imaginary parts of a complex quantity. $\operatorname{diag}(\mathbf{X})$ is used to denote the diagonal elements of the matrix $\mathbf{X}, \bmod (a / b)$ finds the remainder of division of $a$ by $b$, and $\lfloor\cdot\rfloor$ indicates the floor function.

\section{SYSTEM MODEL}

We consider a half-duplex space-division multiple-access (SDMA) SISO cooperative communication system with one source node, $\mathbb{S}, K$ relays, $\mathbb{R}_{1}, \ldots, \mathbb{R}_{K}$, and a single destination node, $\mathbb{D}$, as shown in Fig. 1. Quasi-static and frequency flat-fading channels are considered, which is motivated by prior 


\begin{tabular}{|c|c|c|c|}
\hline $\begin{array}{l}\text { Broadcast } \\
\text { TS to } \\
\text { relays }\end{array}$ & $\begin{array}{c}\text { Broadcast data } \\
\text { to relays }\end{array}$ & $\begin{array}{c}\text { Forward } \\
\text { TS to } \\
\text { D }\end{array}$ & $\begin{array}{c}\text { Forward data to } \\
\mathbb{D}\end{array}$ \\
\hline $\begin{array}{c}\text { Training } \\
\text { Period } \\
\text { (TP) }\end{array}$ & & & $\begin{array}{l}\text { Data Transmission } \\
\text { Period } \\
\text { (DTP) }\end{array}$ \\
\hline
\end{tabular}

Fig. 2. Scheduling diagram for training and data transmission period.

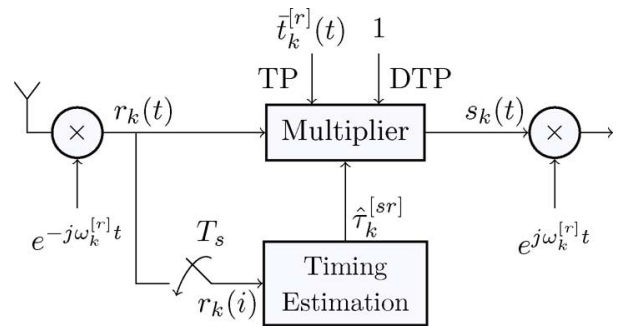

Fig. 3. Block diagram for AF $k$ th Relay Transceiver.

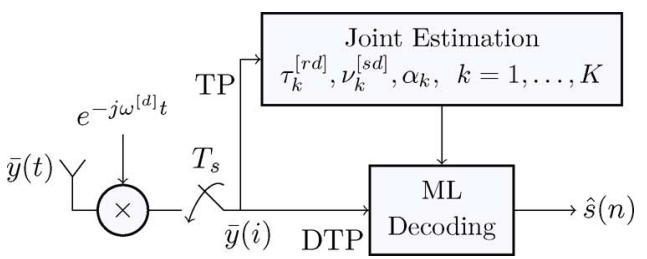

Fig. 4. Block diagram for $\mathrm{AF}$ destination receiver.

research in this field in [6]-[12], [15]. It is assumed that unit amplitude symbols are transmitted from source and relays. The channel gains from $\mathbb{S}$ to $\mathbb{R}_{k}, \mathbb{R}_{k}$ to $\mathbb{D}$, and $\mathbb{S}-\mathbb{R}_{k}-\mathbb{D}$ are denoted by $\rho_{k}, \eta_{k}$, and $\alpha_{k}$, respectively, for $k=\{1, \ldots, K\}$. In Fig. 1, $\tau_{k}$ and $\nu_{k}$ are used to denote timing offsets and CFOs, where superscripts $(\cdot)^{[s r]},(\cdot)^{[r d]}$ and $(\cdot)^{[s d]}$ denote offsets from $\mathbb{S}$ to $\mathbb{R}_{k}, \mathbb{R}_{k}$ to $\mathbb{D}$, and $\mathbb{S}$ to $\mathbb{D}$, respectively. As shown in Fig. 2 , transmission of signals from source to relays to destination consists of a training period (TP) as well as a data transmission period (DTP). Without loss of generality, it is assumed that during the TP, unit-amplitude phase-shift keying (PSK) training signals (TSs) are transmitted to $\mathbb{D}$.

\section{A. AF-Relaying Cooperative Network}

The block diagram for the AF transceiver at $\mathbb{R}_{k}$ and AF receiver at $\mathbb{D}$ are depicted in Figs. 3 and 4, respectively. The proposed training and data transmission methods for the TP and DTP are outlined in the following two subsections.

1) Training Period: The received signal at $\mathbb{R}_{k}$ is down converted by oscillator frequency, $\omega_{k}^{[r]}$, and then over sampled by the factor $Q$. The sampled received signal at the input of the timing estimation block, $r_{k}(i)$ is given by ${ }^{1}$

$r_{k}(i)=\rho_{k} \sum_{n=0}^{L-1} t^{[s]}(n) g\left(i T_{s}-n T-\tau_{k}^{[s r]} T\right) e^{j 2 \pi i \nu_{k}^{[s r]} / Q}$ $+u_{k}(i)$

where $\nu_{k}^{[s r]}$ is the carrier frequency offset, normalized by the symbol duration $T$, between $\mathbb{S}$ and $\mathbb{R}_{k}, \rho_{k}$ denotes the unknown channel gain from $\mathbb{S}$ to $\mathbb{R}_{k}$ that is assumed to not change over a frame but to be distributed as $\mathcal{C N}\left(0, \sigma_{\rho}^{2}\right)$ from frame to frame,

${ }^{1}$ For clarity, we reserve the index $n=\{0, \ldots, L-1\}$ for $T$-spaced samples and index $i=\{0, \ldots, Q L-1\}$ for $T_{s}$-spaced samples. $\tau_{k}^{[s r]}$ is the normalized ${ }^{2}$ fractional unknown timing offset of the sampler at $\mathbb{R}_{k}, T_{s}$ is the sampling time period such that $T_{s}=$ $T / Q, g(t)$ is the transmitter pulse shaping function, $L$ is the length of the source training signal (TS), $t^{[s]}(n)$, and $u_{k}(i)$ is the zero-mean complex baseband additive white Gaussian noise (AWGN) at $\mathbb{R}_{k}$ with variance $\sigma_{u_{k}}^{2}$, i.e., $u_{k}(i) \sim \mathcal{C N}\left(0, \sigma_{u_{k}}^{2}\right)$. Without loss of generality, it is assumed that the noise at all relays have the same variance, i.e., $\sigma_{u}^{2}=\sigma_{u_{1}}^{2}=\cdots=\sigma_{u_{K}}^{2}$.

In order to ensure synchronous transmission and successful cooperation for $\mathrm{AF}$ networks, a timing detector at the $k$ th relay estimates the corresponding timing offset, $\hat{\tau}_{k}^{[s r]}$, using schemes available for point-to-point SISO systems as outlined in [27]. The timing offset estimate $\hat{\tau}_{k}^{[s r]}$ is used as an input to the multiplier to ensure that the $k$ th relay's unit amplitude training signal, $\bar{t}_{k}^{[r]}(t)$, is multiplied by the received signal $r_{k}(t)$ at the appropriate time. The training signal $t_{k}^{[r]}(t)$ used for AF relaying here is given by $\bar{t}_{k}^{[r]}(t)=e^{-j \phi_{k}(n)}$ for $(n-1) T<t<n T$, where $\phi_{k}(n)$ is in between $[-\pi, \pi]$ and denotes the phase of the $n$th symbol of the $k$ th relay's training signal, where $\phi_{k}(n) \neq \phi_{\bar{k}}(n)$, for $k \neq \bar{k}$. The output of the analog multiplier, $s_{k}(t)$, as shown in Fig. 3, is given by

$$
\begin{array}{r}
s_{k}(t)=t_{k}^{[r]}(t) \rho_{k} e^{j 2 \pi \tilde{\nu}_{k}^{[s r]} t} \sum_{n=0}^{L-1} t^{[s]}(n) g\left(t-n T-\epsilon_{k}^{[s r]} T\right) \\
+\bar{t}_{k}^{[r]}(t) u_{k}(t)
\end{array}
$$

where $\epsilon_{k}^{[s r]}=\tau_{k}^{[s r]}-\hat{\tau}_{k}^{[s r]}$ is the timing estimation error between $\mathbb{S}$ and $\mathbb{R}_{k}$ and $\tilde{\nu}_{k}^{[s r]}=\nu_{k}^{[s r]} / T$ is the analog frequency offset between $\mathbb{S}$ and $\mathbb{R}_{k}$. The received signal at $\mathbb{D}$ for AF relaying, $\bar{y}(t)$, is affected by the timing offset from the $k$ th relay to the destination, $\tau_{k}^{[r d]}$, for $k=1 \ldots, K$. Thus, the sampled received signal $\bar{y}(i)$ is given by

$$
\begin{aligned}
\bar{y}(i)= & \sum_{k=1}^{K} \zeta_{k} \eta_{k} s_{k}\left(i T_{s}-\tau_{k}^{[r d]} T\right) e^{j 2 \pi i \nu_{k}^{[r d]} / Q}+w(i) \\
= & \sum_{k=1}^{K} \zeta_{k} \eta_{k} \rho_{k} \bar{t}_{k}^{[r]}\left(i T_{s}-\tau_{k}^{[r d]} T\right) e^{j 2 \pi i \nu_{k}^{[s d]} / Q} \\
& \times \sum_{n=0}^{L-1} t^{[s]}(n) g\left(i T_{s}-n T-\tau_{k}^{[s d]} T\right) \\
& +\sum_{k=1}^{K} \zeta_{k} \eta_{k} u_{k}(i) \bar{t}_{k}^{[r]}\left(i T_{s}-\tau_{k}^{[r d]} T\right) e^{j 2 \pi i \nu_{k}^{[r d]} / Q}+w(i)
\end{aligned}
$$

where

- $\eta_{k}$ denotes the complex unknown channel gain from $\mathbb{R}_{k}$ to $\mathbb{D}$ that is assumed to be distributed as $\mathcal{C N}\left(0, \sigma_{\eta}^{2}\right)$ from frame to frame;

- $\zeta_{k}=1 / \sqrt{\sigma_{\rho}^{2}+\sigma_{u}^{2}}$ satisfies the $k$ th relay's power constraint;

- $\nu_{k}^{[s d]} \triangleq \nu_{k}^{[s r]}+\nu_{k}^{[r d]}$ is the sum of CFOs from $\mathbb{S}-\mathbb{R}_{k}-\mathbb{D}$, $\nu_{k}^{[r d]}$ is the normalized CFO from $\mathbb{R}_{k}$ to $\mathbb{D}^{3}$;

- $\tau_{k}^{[s d]} \triangleq \tau_{k}^{[r d]}+\epsilon_{k}^{[s r]}$,

${ }^{2}$ Throughout this section both the carrier frequency and timing offsets are normalized by $T$.

${ }^{3}$ The constant phase offset $e^{j 2 \pi \nu_{k}^{[s r]} \tau_{k}^{[r d]}}$ due to the timing offset $\tau_{k}^{[r d]}$ is incorporated in the baseband channel $\eta_{k}$. 
- $w(i) \sim \mathcal{C N}\left(0, \sigma_{w}^{2}\right)$ is the AWGN at $\mathbb{D}$; and

- $u_{k}(i)$ has been used in place of $u_{k}\left(i T_{s}-\tau_{k}^{[r d]} T\right)$ since $u_{k}(t)$ denotes the AWGN and its statistics are not affected by the sampling time.

Equation (3) can be written in vector form as shown in (4) at the bottom of the page, where

- $\boldsymbol{\alpha} \triangleq\left[\alpha_{1}, \ldots, \alpha_{K}\right]^{T}$ and $\boldsymbol{\beta} \triangleq\left[\beta_{1}, \ldots, \beta_{K}\right]^{T}$, $\alpha_{k} \triangleq \zeta_{k} \eta_{k} \rho_{k}, \beta_{k} \triangleq \zeta_{k} \eta_{k}$

- $\overline{\mathbf{y}} \triangleq[\bar{y}(0), \bar{y}(1), \ldots, \bar{y}(Q L-1)]^{T}$ is the received signal vector at $\mathbb{D}$;

- $\overline{\mathbf{t}}_{k}^{[r]}\left(\tau_{k}^{[r d]}\right) \quad \triangleq \quad\left[\bar{t}_{k}^{[r]}\left(\tau_{k}^{[r d]} T\right), \bar{t}_{k}^{[r]}(T s\right.$ $\left.\left.\tau_{k}^{[r d]} T\right), \ldots, \bar{t}_{k}^{[r]}\left((Q L-1) T_{s}-\tau_{k}^{[r d]} T\right)\right]^{T}$

- $\overline{\boldsymbol{\Lambda}}_{k} \triangleq \operatorname{diag}\left(\left[e^{j 2 \pi \nu_{k}^{[s d]}}(0) / Q, \ldots, e^{j 2 \pi \nu_{k}^{[s d]}}(Q L-1) / Q\right]\right)$;

- $\boldsymbol{\Lambda}_{k} \triangleq \operatorname{diag}\left(\left[e^{j 2 \pi \nu_{k}^{[r d]}}(0) / Q, \ldots, e^{j 2 \pi \nu_{k}^{[r d]}}(Q L-1) / Q\right]\right)$,

- $\left[\overline{\mathbf{G}}_{k}\right]_{m, \ell} \triangleq g\left(m T_{s}-\ell T-\tau_{k}^{[s d]} T\right)$ is a $Q L \times L$ matrix;

- $\mathbf{t}^{[s]} \triangleq\left[t^{[s]}(0), \ldots, t^{[s]}(L-1)\right]^{T}$

- $\mathbf{w} \triangleq[w(0), \ldots, w(Q L-1)]^{T} ;$ and

- $\mathbf{v}_{k} \triangleq\left[v_{k}(0), \ldots, v_{k}(Q L-1)\right]^{T}$, and $v_{k}(i) \triangleq$ $u_{k}(i) \bar{t}_{k}^{[r]}\left(i T_{s}-\tau_{k}^{[r d]} T\right)$.

Note that $v_{k}(i)$ has the same statistical properties as $u_{k}(i)$, for $i=\{0, \ldots, Q L-1\}$, due to the assumption of unit-amplitude training signals.

Remark 1: Unlike [6], the proposed processing structure at the relays in Fig. 3 is not based on the assumption of perfect timing and frequency offset estimation and matched filtering at the relays [28]. In fact, [28] shows that the signal model in [6] does not resemble that of AF relaying. More importantly, in a follow-up paper, the authors of [6] confirm that further research is needed to enable perfect timing synchronization in practical AF-relaying cooperative networks [29]. In order to address this issue, we have proposed a novel relaying structure that does not require perfect timing offset estimation and matched-filtering at the relays. In addition, in our proposed model, the relays do not perform frequency offset and channel estimation during the TP.

2) Data Transmission Period: Modulated data symbol vector $\mathbf{s} \triangleq[s(0), s(1), \ldots, s(L-1)]^{T}$ is transmitted from $\mathbb{S}$ to the relays. As shown in Fig. 3, after performing timing correction using timing offset estimates obtained in the TP, $\mathbb{R}_{k}$ forwards the received signal to $\mathbb{D}$. The received signal at $\mathbb{D}$ in the DTP can be written as ${ }^{4}$

$$
\overline{\mathbf{y}}=\sum_{k=1}^{K} \alpha_{k} \overline{\mathbf{\Lambda}}_{k} \overline{\mathbf{G}}_{k} \mathbf{s}+\sum_{k=1}^{K} \beta_{k} \boldsymbol{\Lambda}_{k} \mathbf{u}_{k}+\mathbf{w}
$$

where $\mathbf{u}_{k} \triangleq\left[u_{k}(0), \ldots, u_{k}(Q L-1)\right]^{T}$. Fig. 4 summarizes the proposed transceiver structure at $\mathbb{D}$ for AF relaying.

\section{B. DF-Relaying Cooperative Network}

The block diagram for the DF transceiver at $\mathbb{R}_{k}$ is shown in Fig. 5. The receiver structure at $\mathbb{D}$ is similar to that of $A F$

\footnotetext{
${ }^{4}$ The matrix $\overline{\mathbf{G}}_{k}$ takes into account the timing offset estimation error from $\mathbb{S}$ to $\mathbb{R}_{k}$ and the timing offset from $\mathbb{P}_{k}$ to $\mathbb{D}$.
}

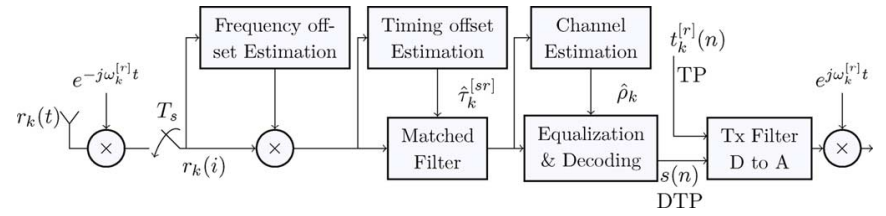

Fig. 5. Block diagram for DF transceiver at the $k$ th relay.

relaying in Fig. 4 with the exception that the received signal is represented by $y(t)$ instead of $\bar{y}(t)$.

1) Training Period: Similar to AF, the sampled received signal at $\mathbb{R}_{k}, r_{k}(i)$, is given by (1). However, in the case of DF relaying the received signal at $\mathbb{R}_{k}$ needs to be decoded. Therefore, during the TP, $\hat{\rho}_{k}, \hat{\tau}_{k}^{[s r]}$, and $\hat{\nu}_{k}^{[s r]}$ are jointly estimated and compensated using conventional schemes for SISO point-topoint systems [27]. Subsequently, $\mathbb{R}_{k}$ forwards its known distinct TS, $\mathbf{t}_{k}^{[r]} \triangleq\left[t_{k}^{[r]}(0), \ldots, t_{k}^{[r]}(L-1)\right]^{T}$ to $\mathbb{D}$. The received signal at $\mathbb{D}, \mathbf{y} \triangleq[y(0), \ldots, y(Q L-1)]^{T}$, is given by

$$
\mathbf{y}=\underbrace{\left[\boldsymbol{\Lambda}_{1} \mathbf{G}_{1} \mathbf{t}_{1}^{[r]}, \ldots, \boldsymbol{\Lambda}_{K} \mathbf{G}_{K} \mathbf{t}_{K}^{[r]}\right]}_{\triangleq \boldsymbol{\Omega}} \boldsymbol{\eta}+\mathbf{w}
$$

where $\boldsymbol{\eta} \triangleq\left[\eta_{1}, \ldots, \eta_{K}\right]^{T}, \tau_{k}^{[r d]}$, is the normalized fractional unknown timing offset between $\mathbb{R}_{k}$ and $\mathbb{D}, \boldsymbol{\Lambda}_{k}$ is defined below (4), and $\left[\mathbf{G}_{k}\right]_{m, \ell} \triangleq g\left(m T_{s}-\ell T-\tau_{k}^{[r d]} T\right)$ is a $Q L \times L$ matrix. Note that the notation, $\mathbf{t}_{k}^{[r]}$ is used instead of $\overline{\mathbf{t}}_{k}^{[r]}$ to distinguish between the TSs for DF and AF relaying.

2) Data Transmission Period: For DF relaying, it is assumed that cyclic redundancy checks (CRC) are applied at the relays to ensure that the relays only forward correctly decoded signals to $\mathbb{D}$. Subsequently, the received signal at $\mathbb{D}$ in the DTP is given by

$$
\mathbf{y}=\sum_{k=1}^{K} \eta_{k} \boldsymbol{\Lambda}_{k} \mathbf{G}_{k} \mathbf{s}+\mathbf{w} .
$$

The receiver structure at $\mathbb{D}$ is given by Fig. 4 with the exception that $\tau_{k}^{[r d]}, \nu_{k}^{[r d]}$, and $\eta_{k}$ are replaced by $\tau_{k}^{[s d]}, \nu_{k}^{[s d]}$, and $\alpha_{k}$, for $k=\{1, \ldots, K\}$, respectively.

\section{CRAMER-RAO LOWER BOUND}

In this section, new exact closed-form CRLBs for joint estimation of multiple channel gains, MCFOs, and MTOs for AF-relaying cooperative networks are derived. For the case of DF relaying, we extend the results in [17] and present closed-form CRLBs for this multiple parameter estimation problem.

\section{A. CRLB for AF Relaying}

Throughout this section, it is assumed that the forwarded AWGN from the relays, $\mathbb{R}_{k}$ and $\mathbb{R}_{m}, \mathbf{v}_{k}$ and $\mathbf{v}_{m}$, respectively, $\forall k \neq m$, and the AWGN at $\mathbb{D}, \mathbf{w}$, are mutually independent. Accordingly, the received signal vector at $\mathbb{D}, \overline{\mathbf{y}}$ in (4), is a

$$
\overline{\mathbf{y}}=\underbrace{\left[\left(\overline{\boldsymbol{\Lambda}}_{1} \overline{\mathbf{G}}_{1} \mathbf{t}^{[s]}\right) \odot \overline{\mathbf{t}}_{1}^{[r]}\left(\tau_{k}^{[r d]}\right), \ldots,\left(\overline{\boldsymbol{\Lambda}}_{K} \overline{\mathbf{G}}_{K} \mathbf{t}^{[s]}\right) \odot \overline{\mathbf{t}}_{K}^{[r]}\left(\tau_{k}^{[r d]}\right)\right]}_{\triangleq \overline{\boldsymbol{\Omega}}} \boldsymbol{\alpha}+\underbrace{\left[\boldsymbol{\Lambda}_{1} \mathbf{v}_{1}, \cdots, \boldsymbol{\Lambda}_{K} \mathbf{v}_{K}\right]}_{\triangleq \overline{\mathbf{\Psi}}} \boldsymbol{\beta}+\mathbf{w}
$$


circularly symmetric complex Gaussian random variable, i.e., $\overline{\mathbf{y}} \sim \mathcal{C} \mathcal{N}\left(\boldsymbol{\mu}_{\overline{\mathbf{y}}}, \boldsymbol{\Sigma}_{\overline{\mathbf{y}}}\right)$, with mean $\boldsymbol{\mu}_{\overline{\mathbf{y}}}=\overline{\boldsymbol{\Omega}} \boldsymbol{\alpha}$ and covariance matrix $\boldsymbol{\Sigma}_{\overline{\mathbf{y}}}=\left(\sigma_{u}^{2} \sum_{k=1}^{K}\left|\beta_{k}\right|^{2}+\sigma_{w}^{2}\right) \mathbf{I}_{L Q}$, where $\mathbf{I}_{L Q}$ is the identity matrix of size $L Q \times L Q\left(\Sigma_{\overline{\mathbf{y}}}\right.$ is derived in Appendix A). As discussed in [8] and [16], in the case of AF relaying, only the overall channel gains, $\boldsymbol{\alpha}$, need to be estimated. As a result, the parameter vector of interest for AF relaying, $\overline{\boldsymbol{\theta}}$, is given by

$$
\overline{\boldsymbol{\theta}} \triangleq\left[\Re\{\boldsymbol{\alpha}\}^{T}, \Im\{\boldsymbol{\alpha}\}^{T}, \overline{\boldsymbol{\nu}}^{T}, \overline{\boldsymbol{\tau}}^{T}\right]^{T}
$$

where $\overline{\boldsymbol{\nu}} \triangleq\left[\bar{\nu}_{1}, \ldots, \bar{\nu}_{K}\right]^{T}$ and $\overline{\boldsymbol{\tau}} \triangleq\left[\bar{\tau}_{1}, \ldots, \bar{\tau}_{K}\right]^{T}$ and for notational simplicity, $\bar{\nu}_{k}$ and $\bar{\tau}_{k}$ are used to denote $\nu_{k}^{[s d]}$ and $\tau_{k}^{[r d]}$ for AF relaying, respectively.

Proposition 1: Based on the proposed training method, the Fisher's information matrix (FIM) for the estimation of $\overline{\boldsymbol{\theta}}$ is given by (9) shown at the bottom of the page, where

- $\sigma_{n}^{2} \triangleq \sigma_{u}^{2} \sum_{k=1}^{K}\left|\beta_{k}\right|^{2}+\sigma_{w}^{2}$;

- $\overline{\boldsymbol{\Gamma}} \triangleq\left[\left(\overline{\boldsymbol{\Lambda}}_{1} \overline{\mathbf{R}}_{1} \mathbf{t}^{[s]}\right) \odot \overline{\mathbf{t}}_{1}^{[r]}\left(\tau_{k}^{[r d]}\right), \cdots,\left(\overline{\boldsymbol{\Lambda}}_{K} \overline{\mathbf{R}}_{K} \mathbf{t}^{[s]}\right) \odot\right.$ $\left.\overline{\mathbf{t}}_{K}^{[r]}\left(\tau_{k}^{[r d]}\right)\right]$;

- $\mathbf{D} \triangleq 2 \pi / Q \times \operatorname{diag}([0,1, \ldots, L Q-1])$;

- $\overline{\mathbf{H}} \triangleq \operatorname{diag}\left(\alpha_{1}, \ldots, \alpha_{K}\right)$; and

- $\overline{\mathbf{R}}_{k} \triangleq \partial \overline{\mathbf{G}}_{k} / \partial \bar{\tau}_{k}$.

Proof: See Appendix A.

Let us rewrite (9) as

$$
\mathbf{F}^{\mathrm{AF}} \triangleq \frac{2}{\sigma_{n}^{2}}\left[\begin{array}{cc}
\overline{\mathbf{F}}_{11} & \overline{\mathbf{Z}} \\
\overline{\mathbf{Z}}^{T} & \overline{\mathbf{F}}_{22}
\end{array}\right]
$$

where $\overline{\mathbf{F}}_{11}$ and $\overline{\mathbf{F}}_{22}$ are the upper left and lower right $2 K \times 2 K$ submatrices of $\overline{\mathcal{F}}$, respectively, and $\overline{\mathbf{Z}}$ is the upper right $2 K \times$ $2 K$ submatrix of $\overline{\mathcal{F}}$. Using partitioned matrix inverse [8], [30], the closed-form CRLB for the estimation of $\overline{\boldsymbol{\theta}}$ can be determined as shown in (11) at the bottom of the page. Similarly, $\overline{\mathbf{F}}_{11}^{-1}$ and $\overline{\mathbf{F}}_{22}^{-1}$ are found as

$$
\overline{\mathbf{F}}_{11}^{-1}=\left[\begin{array}{cc}
\Re\left\{\left(\overline{\mathbf{\Omega}}^{H} \overline{\mathbf{\Omega}}\right)^{-1}\right\} & -\Im\left\{\left(\overline{\mathbf{\Omega}}^{H} \overline{\mathbf{\Omega}}\right)^{-1}\right\} \\
\Im\left\{\left(\overline{\mathbf{\Omega}}^{H} \overline{\mathbf{\Omega}}\right)^{-1}\right\} & \Re\left\{\left(\overline{\mathbf{\Omega}}^{H} \overline{\mathbf{\Omega}}\right)^{-1}\right\}
\end{array}\right]
$$

and (13) shown at the bottom of the page.

The CRLB for the estimation of MCFOs and MTOs is given by diagonal elements of the matrix, $\bar{\Upsilon}$ in (11), and can be written in closed-form as

$$
\begin{aligned}
\operatorname{CRLB}(\overline{\boldsymbol{\nu}}, \overline{\boldsymbol{\tau}}) \\
=\frac{\sigma_{n}^{2}}{2} \operatorname{diag}\left(\left(\overline{\mathbf{F}}_{22}-\overline{\mathbf{Z}}^{T} \overline{\mathbf{F}}_{11}^{-1} \overline{\mathbf{Z}}\right)^{-1}\right) \\
=\frac{\sigma_{n}^{2}}{2} \operatorname{diag}\left(\overline{\mathbf{F}}_{22}^{-1}+\overline{\mathbf{F}}_{22}^{-1} \overline{\mathbf{Z}}^{T}\left(\overline{\mathbf{F}}_{11}-\overline{\mathbf{Z}} \overline{\mathbf{F}}_{22}^{-1} \overline{\mathbf{Z}}^{T}\right)^{-1}\right. \\
\left.\quad \times \overline{\mathbf{Z}} \overline{\mathbf{F}}_{22}^{-1}\right)
\end{aligned}
$$

where the second equality in (14) follows from the matrix inverse identity $\left(\mathbf{A}-\mathbf{C}^{T} \mathbf{D}^{-1} \mathbf{C}\right)^{-1}=\mathbf{A}^{-1}+\mathbf{A}^{-1} \mathbf{C}^{T}(\mathbf{D}-$ $\left.\mathbf{C A}^{-1} \mathbf{C}^{T}\right)^{-1} \mathbf{C A}^{-1}$ for invertible matrices $\mathbf{A}$ and $\mathbf{D}$ in [30]. In this case, $\overline{\mathbf{F}}_{22}, \overline{\mathbf{F}}_{11}$, and $\overline{\mathbf{Z}}$ in (14) correspond to $\mathbf{A}, \mathbf{D}$, and C, respectively. Similarly, the CRLB for the estimation of combined real and imaginary parts of $\boldsymbol{\alpha}$ is derived as

$$
\begin{aligned}
& \operatorname{CRLB}(\Re\{\boldsymbol{\alpha}\}, \Im\{\boldsymbol{\alpha}\}) \\
& \quad=\frac{\sigma_{n}^{2}}{2} \operatorname{diag}\left(\overline{\mathbf{F}}_{11}^{-1}+\overline{\mathbf{F}}_{11}^{-1} \overline{\mathbf{Z}} \bar{\Upsilon} \overline{\mathbf{Z}}^{T} \overline{\mathbf{F}}_{11}^{-1}\right) . \\
& \mathbf{C R L B}(\boldsymbol{\alpha}) \\
& \quad=\frac{\sigma_{n}^{2}}{2} \operatorname{diag}\left(\mathbf{B} \overline{\mathbf{F}}_{11}^{-1} \mathbf{B}^{H}+\mathbf{B} \overline{\mathbf{F}}_{11}^{-1} \overline{\mathbf{Z}} \bar{\Upsilon} \overline{\mathbf{Z}}^{T} \overline{\mathbf{F}}_{11}^{-1} \mathbf{B}^{H}\right)
\end{aligned}
$$

where $\mathbf{B} \equiv\left[\begin{array}{ll}\mathbf{I}_{K} & j \mathbf{I}_{K}\end{array}\right]$ is used to obtain the CRLB of $\boldsymbol{\alpha}$ according to [31].

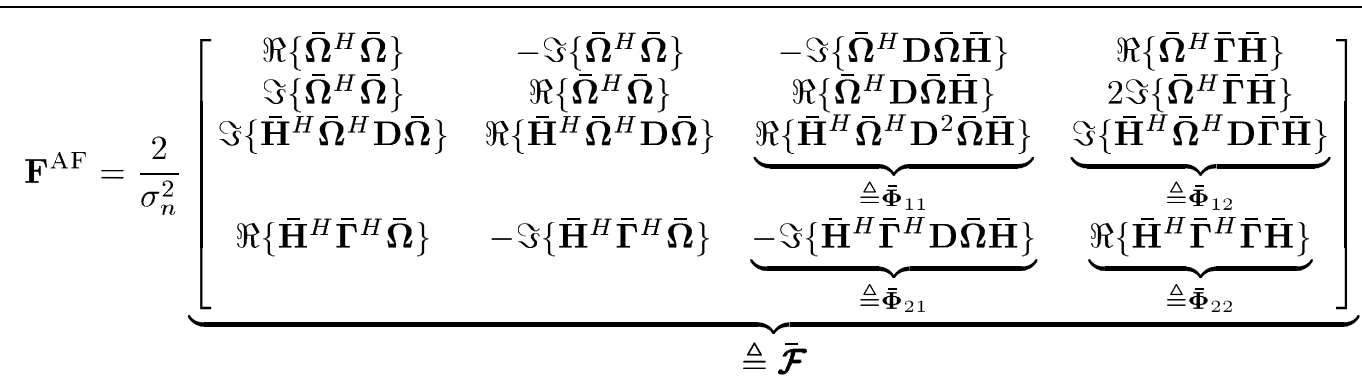

$$
\begin{aligned}
& \operatorname{CRLB}(\overline{\boldsymbol{\theta}})=\frac{\sigma_{n}^{2}}{2}(\left[\begin{array}{cc}
\overline{\mathbf{F}}_{11}^{-1} & \mathbf{0} \\
\mathbf{0} & \mathbf{0}
\end{array}\right]+\left[\begin{array}{c}
-\overline{\mathbf{F}}_{11}^{-1} \overline{\mathbf{Z}} \\
\mathbf{I}
\end{array}\right] \underbrace{\left[\overline{\mathbf{F}}_{22}-\overline{\mathbf{Z}}^{T} \overline{\mathbf{F}}_{11}^{-1} \overline{\mathbf{Z}}\right]^{-1}}_{\triangleq \overline{\mathbf{Y}}}\left[-\overline{\mathbf{Z}}^{T} \overline{\mathbf{F}}_{11}^{-1} \quad \mathbf{I}\right]) \\
& =\frac{\sigma_{n}^{2}}{2}\left[\begin{array}{cc}
\overline{\mathbf{F}}_{11}^{-1}+\overline{\mathbf{F}}_{11}^{-1} \overline{\mathbf{Z}} \bar{\Upsilon} \overline{\mathbf{Z}}^{T} \overline{\mathbf{F}}_{11}^{-1} & -\overline{\mathbf{F}}_{11}^{-1} \overline{\mathbf{Z}} \overline{\mathbf{\Upsilon}} \\
-\bar{\Upsilon}^{T} \overline{\mathbf{Z}}^{T} \overline{\mathbf{F}}_{11}^{-1} & \boldsymbol{\Upsilon} \mathbf{\Upsilon}
\end{array}\right]
\end{aligned}
$$

$$
\overline{\mathbf{F}}_{22}^{-1}=\left[\begin{array}{cc}
\left(\overline{\mathbf{\Phi}}_{11}-\overline{\mathbf{\Phi}}_{12} \overline{\mathbf{\Phi}}_{22}^{-1} \overline{\mathbf{\Phi}}_{21}\right)^{-1} & -\overline{\mathbf{\Phi}}_{11}^{-1} \overline{\mathbf{\Phi}}_{12}\left(\overline{\mathbf{\Phi}}_{22}-\overline{\mathbf{\Phi}}_{21} \overline{\mathbf{\Phi}}_{11}^{-1} \overline{\mathbf{\Phi}}_{12}\right)^{-1} \\
-\overline{\mathbf{\Phi}}_{22}^{-1} \overline{\mathbf{\Phi}}_{21}\left(\overline{\mathbf{\Phi}}_{11}-\overline{\mathbf{\Phi}}_{12} \overline{\mathbf{\Phi}}_{22}^{-1} \overline{\mathbf{\Phi}}_{21}\right)^{-1} & \left(\overline{\mathbf{\Phi}}_{22}-\overline{\mathbf{\Phi}}_{21} \overline{\mathbf{\Phi}}_{11}^{-1} \overline{\mathbf{\Phi}}_{12}\right)^{-1}
\end{array}\right] .
$$




\section{B. CRLB for DF Relaying}

Similar to the case of AF relaying, the received signal vector in (6), $\mathbf{y} \sim \mathcal{C N}\left(\boldsymbol{\mu}_{\mathbf{y}}, \boldsymbol{\Sigma}_{\mathbf{y}}\right)$, with mean $\boldsymbol{\mu}_{\mathbf{y}}=\boldsymbol{\Omega} \boldsymbol{\eta}$ and covariance matrix $\boldsymbol{\Sigma}_{\mathbf{y}}=\sigma_{w}^{2} \mathbf{I}_{L Q}$. The parameter vector of interest for DF relaying, $\boldsymbol{\theta}$, is given by

$$
\boldsymbol{\theta} \triangleq\left[\Re\{\boldsymbol{\eta}\}^{T}, \Im\{\boldsymbol{\eta}\}^{T}, \boldsymbol{\nu}^{T}, \boldsymbol{\tau}^{T}\right]^{T}
$$

where $\boldsymbol{\nu} \triangleq\left[\nu_{1}, \ldots, \nu_{K}\right]^{T}$ and $\boldsymbol{\tau} \triangleq\left[\tau_{1}, \ldots, \tau_{K}\right]^{T}$ and for notational simplicity, $\nu_{k}$ and $\tau_{k}$ are used to denote $\nu_{k}^{[r d]}$ and $\tau_{k}^{[r d]}$, respectively. The FIM for the estimation of $\boldsymbol{\theta}[17]$ is given by (17) shown at the bottom of the page, where $\boldsymbol{\Gamma} \triangleq\left[\boldsymbol{\Lambda}_{1} \mathbf{R}_{1} \mathbf{t}_{1}^{[r]}, \ldots, \boldsymbol{\Lambda}_{K} \mathbf{R}_{K} \mathbf{t}_{K}^{[r]}\right], \mathbf{H} \triangleq \operatorname{diag}\left\{\eta_{1}, \ldots, \eta_{K}\right\}$, and $\mathbf{R}_{k} \triangleq \partial \mathbf{G}_{k} / \partial \tau_{k}$. Let us rewrite (17) as

$$
\mathbf{F}^{\mathrm{DF}} \triangleq \frac{2}{\sigma_{w}^{2}}\left[\begin{array}{cc}
\mathbf{F}_{11} & \mathbf{Z} \\
\mathbf{Z}^{T} & \mathbf{F}_{22}
\end{array}\right]
$$

where $\mathbf{F}_{11}$ and $\mathbf{F}_{22}$ denote the upper left and lower right $2 K \times$ $2 K$ submatrices of $\mathcal{F}$, respectively, and $\mathbf{Z}$ is the upper right $2 K \times 2 K$ submatrix of $\mathcal{F}$. Using similar steps as that of $\mathrm{AF}$ relaying, the closed-form CRLB for the estimation of $\boldsymbol{\theta}$ can be determined as

$$
\operatorname{CRLB}(\boldsymbol{\theta})=\frac{\sigma_{w}^{2}}{2}\left[\begin{array}{cc}
\mathbf{F}_{11}^{-1}+\mathbf{F}_{11}^{-1} \mathbf{Z} \Upsilon \mathbf{Z}^{T} \mathbf{F}_{11}^{-1} & -\mathbf{F}_{11}^{-1} \mathbf{Z} \Upsilon \\
-\mathbf{\Upsilon} \mathbf{Z}^{T} \mathbf{F}_{11}^{-1} & \mathbf{\Upsilon}
\end{array}\right]
$$

where $\mathbf{\Upsilon} \triangleq\left[\mathbf{F}_{22}-\mathbf{Z}^{T} \mathbf{F}_{11}^{-1} \mathbf{Z}\right]^{-1}$. Moreover, $\mathbf{F}_{11}^{-1}$ and $\mathbf{F}_{22}^{-1}$ are given by replacing $\overline{\boldsymbol{\Omega}}$ and $\overline{\mathbf{\Phi}}_{l, m}$ with $\boldsymbol{\Omega}$ and $\boldsymbol{\Phi}_{l, m}, \forall l, m$ in (12) and (13), respectively. Using (18) the closed-form CRLBs for the estimation of $\boldsymbol{\nu}, \boldsymbol{\tau}$, and $\boldsymbol{\eta}$ can be determined as

$$
\begin{aligned}
& \operatorname{CRLB}(\boldsymbol{\nu}, \boldsymbol{\tau}) \\
& \quad=\frac{\sigma_{w}^{2}}{2} \operatorname{diag}\left(\mathbf{F}_{22}^{-1}+\mathbf{F}_{22}^{-1} \mathbf{Z}^{T}\left(\mathbf{F}_{11}-\mathbf{Z F}_{22}^{-1} \mathbf{Z}^{T}\right)^{-1}\right. \\
& \left.\quad \times \mathbf{Z} \mathbf{F}_{22}^{-1}\right) \\
& \mathbf{C R L B}(\boldsymbol{\eta}) \\
& =\frac{\sigma_{w}^{2}}{2}\left(\mathbf{B F}_{11}^{-1} \mathbf{B}^{H}+\mathbf{B} \mathbf{F}_{11}^{-1} \mathbf{Z} \Upsilon \mathbf{Z}^{T} \mathbf{F}_{11}^{-1} \mathbf{B}^{H}\right)
\end{aligned}
$$

The following remarks are in order:

Remark 2: Equations (9) and (11) for AF relaying, and (17) and (18) for DF-relaying cooperative networks demonstrate that for both choices of protocols, the FIM and the CRLB for estimation of MCFOs, MTOs, and channel gains are not block diagonal. Thus, there exists coupling between the estimation errors of MCFOs, MTOs, and channel gains. This shows the importance of jointly estimating MCFOs, MTOs, and channel gains in multi-relay cooperative networks. More importantly, this result indicates that the previously proposed methods that assume perfect frequency or timing synchronization while estimating
MCFOs and MTOs in [6] and [8], respectively, cannot be applied to estimate MCFOs, MTOs, and multiple channel gains in distributed $\mathrm{AF}$ and $\mathrm{DF}$ cooperative networks.

Remark 3: The CRLBs for the estimation of $\overline{\boldsymbol{\nu}}, \overline{\boldsymbol{\tau}}$ and $\boldsymbol{\alpha}$ for AF relaying in (14) and (15b) depend on source-relay-destination carrier frequency offsets, $\left\{\bar{\nu}_{k}\right\}_{k=1}^{K}$, timing offsets, $\left\{\bar{\tau}_{k}\right\}_{k=1}^{K}$, and channel gains, $\left\{\alpha_{k}\right\}_{k=1}^{K}$, through the matrices $\overline{\mathbf{F}}_{11}$ and $\mathbf{Z}$ in (10). As anticipated, the CRLBs in (14) and (15b) are also dependent on the TS length and the choice of the TSs broadcasted from both $\mathbb{S}$ and the relays. Based on the CRLBs in (19a) and (19b), similar dependencies can be also deduced for the case of DF relaying. Therefore, we can conclude that the choice of TS and its length are important design parameters that significantly impact the performance of MCFO, MTO, and channel estimators in distributed multi-relay cooperative networks. Optimal TS design for estimation of MCFOs or MTOs is addressed in [9] and [7], respectively. However, to the best of authors' knowledge the design of TSs for joint MCFO and MTO estimation is still an open area of research.

\section{JOINT PARAMETER ESTIMATION}

In this section, in order to reduce the computational complexity associated with the estimation of MCFOs, MTOs, and multiple channel gains for DF-and AF-relaying networks, two iterative estimators are proposed based on the ECM and SAGE algorithms and their computational complexity is analyzed.

\section{A. Estimation Algorithms for DF Cooperative Networks}

We first outline the ML estimator due to its desirable asymptotic properties [31]. The ML estimator presented here is also required to initialize the proposed ECM and SAGE algorithms.

1) ML Estimator for DF Relaying: Based on the signal model in (6), the ML estimates of $\boldsymbol{\nu}, \boldsymbol{\tau}$, and $\boldsymbol{\eta}$ are given by [17]

$$
\begin{aligned}
\hat{\boldsymbol{\nu}}, \hat{\boldsymbol{\tau}} & =\arg \max _{\boldsymbol{\nu}, \boldsymbol{\tau}} \mathbf{y}^{H} \boldsymbol{\Omega}\left(\boldsymbol{\Omega}^{H} \boldsymbol{\Omega}\right)^{-1} \boldsymbol{\Omega}^{H} \mathbf{y} \\
\hat{\boldsymbol{\eta}} & =\left(\boldsymbol{\Omega}^{H} \boldsymbol{\Omega}\right)^{-1} \boldsymbol{\Omega}^{H} \mathbf{y} .
\end{aligned}
$$

Alternating projection (AP) is used to reduce the dimensionality of the maximization problem in (20) into a series of one-dimensional searches [32]. Even though AP is not guaranteed to converge to the true estimates, the results in [32, Section IV.A], demonstrate that AP always converges to a local maximum and through proper initialization, AP results in global convergence. Following the ML-based approach in [22], $\hat{\nu}_{k}$ and $\hat{\tau}_{k}$ are initialized to $0, \forall, k$ in our simulations. In addition, the numerical simulations in Fig. 6 indicate that using the above initialization, AP converges to the true timing and frequency offset estimates in two cycles only.

$$
\mathbf{F}^{\mathrm{DF}}=\frac{2}{\sigma_{w}^{2}} \underbrace{\left[\begin{array}{cccc}
\Re\left\{\boldsymbol{\Omega}^{H} \boldsymbol{\Omega}\right\} & -\Im\left\{\boldsymbol{\Omega}^{H} \boldsymbol{\Omega}\right\} & -\Im\left\{\boldsymbol{\Omega}^{H} \mathbf{D} \boldsymbol{\Omega} \mathbf{H}\right\} & \Re\left\{\boldsymbol{\Omega}^{H} \boldsymbol{\Gamma} \mathbf{H}\right\} \\
\Im\left\{\boldsymbol{\Omega}^{H} \boldsymbol{\Omega}\right\} & \Re\left\{\boldsymbol{\Omega}^{H} \boldsymbol{\Omega}\right\} & \Re\left\{\boldsymbol{\Omega}^{H} \mathbf{D} \boldsymbol{\Omega} \mathbf{H}\right\} & \Im\left\{\boldsymbol{\Omega}^{H} \boldsymbol{\Gamma} \mathbf{H}\right\} \\
\Im\left\{\mathbf{H}^{H} \boldsymbol{\Omega}^{H} \mathbf{D} \boldsymbol{\Omega}\right\} & \Re\left\{\mathbf{H}^{H} \boldsymbol{\Omega}^{H} \mathbf{D} \boldsymbol{\Omega}\right\} & \underbrace{\Re\left\{\mathbf{H}^{H} \boldsymbol{\Omega}^{H} \mathbf{D}^{2} \boldsymbol{\Omega} \mathbf{H}\right\}}_{\triangleq \boldsymbol{\Phi}_{11}} & \underbrace{\Im\left\{\mathbf{H}^{H} \boldsymbol{\Omega}^{H} \mathbf{D} \boldsymbol{\Gamma} \mathbf{H}\right\}}_{\triangleq \boldsymbol{\Phi}_{21}} \\
\Re\left\{\mathbf{H}^{H} \boldsymbol{\Gamma}^{H} \boldsymbol{\Omega}\right\} & -\Im\left\{\mathbf{H}^{H} \boldsymbol{\Gamma}^{H} \boldsymbol{\Omega}\right\} & \underbrace{-\Im\left\{\mathbf{H}^{H} \boldsymbol{\Gamma}^{H} \mathbf{D} \boldsymbol{\Omega} \mathbf{H}\right\}}_{\triangleq \boldsymbol{\Phi}_{12}} & \underbrace{\Re\left\{\mathbf{H}^{H} \boldsymbol{\Gamma}^{H} \boldsymbol{\Gamma} \mathbf{H}\right\}}_{\triangleq \boldsymbol{\Phi}_{22}}
\end{array}\right]}_{\triangleq \mathcal{F}}
$$


2) ECM Estimator for DF Relaying: The entries in the vector $\boldsymbol{\theta}$ in (16) can be rearranged into the new parameter vector $\boldsymbol{\lambda} \triangleq\left[\boldsymbol{\lambda}_{1}^{T}, \ldots, \boldsymbol{\lambda}_{K}^{T}\right]^{T}$, where $\boldsymbol{\lambda}_{k} \triangleq\left[\nu_{k}, \tau_{k}, \eta_{k}\right]^{T}$, for $k=\{1, \ldots, K\}$. In the expectation-maximization (EM) terminology, the received training signal $\mathbf{y}$ in (6) represents the incomplete data set [33]. Following [34], we define the hidden or complete data set as $\mathbf{z} \triangleq\left[\mathbf{z}_{1}^{T}, \ldots, \mathbf{z}_{K}^{T}\right]^{T}$, with

$$
\mathbf{z}_{k}=\eta_{k} \boldsymbol{\Lambda}_{k} \mathbf{G}_{k} \mathbf{t}_{k}^{[r]}+\mathbf{w}_{k}
$$

where $\mathbf{w}_{k} \sim \mathcal{C N}\left(0, \gamma_{k} \sigma_{w}^{2} \mathbf{I}_{L Q}\right)$ with $\sum_{k=1}^{K} \gamma_{k}=1$ and $\mathbf{z}_{k}$ is the hidden variable. In (21), $\mathbf{w}_{k}$ is obtained by decomposing the total noise vector w into $K$ components such that $\sum_{k=1}^{K} \mathbf{w}_{k}=\mathbf{w}$ where $\gamma_{k}$ may be chosen such that $\gamma_{k}=1 / K$, $\forall k$ [35]. Based on (21), the relationship between the complete and incomplete data sets is given by $\sum_{k=1}^{K} \mathbf{z}_{k}=\mathbf{y}$. In order to indicate the iterative processing let us define $\hat{\boldsymbol{\lambda}}^{[m]} \triangleq\left[\left(\hat{\boldsymbol{\lambda}}_{1}^{[m]}\right)^{T}, \ldots,\left(\hat{\boldsymbol{\lambda}}_{K}^{[m]}\right)^{T}\right]^{T}$ as the estimated value of $\boldsymbol{\lambda}$ at the $m$ th iteration, where $\hat{\boldsymbol{\lambda}}_{k}^{[m]} \triangleq\left[\hat{\nu}_{k}^{[m]}, \hat{\tau}_{k}^{[m]}, \hat{\eta}_{k}^{[m]}\right]^{T}$. Note that rough initial estimates, $\hat{\nu}_{k}^{[0]}, \hat{\tau}_{k}^{[0]}, \hat{\eta}_{k}^{[0]}$, may be obtained using alternating projection via (20) using a coarse step size. The E-Step and $M$-Step of the proposed ECM algorithms at the $m$ th iteration are derived next.

a) E-Step: Using the received signal $\mathbf{y}$ and the current estimates, $\hat{\boldsymbol{\lambda}}^{[m]}$, we compute the expectation of the log-likelihood function (LLF) of the complete data space given the parameter $\boldsymbol{\lambda}, \mathbf{N}\left(\boldsymbol{\lambda} \mid \hat{\boldsymbol{\lambda}}^{[m]}\right)$. That is

$$
\mathbf{N}\left(\boldsymbol{\lambda} \mid \hat{\boldsymbol{\lambda}}^{[m]}\right) \triangleq \mathbb{E}\left\{\log f(\mathbf{z} \mid \boldsymbol{\lambda}) \quad \mid \mathbf{y}, \hat{\boldsymbol{\lambda}}^{[m]}\right\}
$$

where the probability density function of $\mathbf{z}$ given $\boldsymbol{\lambda}$ is determined as

$$
\begin{aligned}
f(\mathbf{z} \mid \boldsymbol{\lambda}) & =\prod_{k=1}^{K} f\left(\mathbf{z}_{k} \mid \boldsymbol{\lambda}_{k}\right) \\
& =\prod_{k=1}^{K} \frac{1}{\left(\pi \gamma_{k} \sigma_{w}^{2}\right)^{L Q}} \exp \left\{\frac{\left\|\mathbf{z}_{k}-\eta_{k} \boldsymbol{\Lambda}_{k} \mathbf{G}_{k} \mathbf{t}_{k}^{[r]}\right\|^{2}}{\gamma_{k} \sigma_{w}^{2}}\right\} .
\end{aligned}
$$

Substituting (23) into (22), we obtain

$$
\begin{aligned}
\mathbf{N} & \left(\boldsymbol{\lambda} \mid \hat{\boldsymbol{\lambda}}^{[m]}\right) \\
& =E_{1}-\mathbb{E}\left\{\sum_{k=1}^{K} \frac{1}{\gamma_{k} \sigma_{w}^{2}}\left\|\mathbf{z}_{k}-\eta_{k} \boldsymbol{\Lambda}_{k} \mathbf{G}_{k} \mathbf{t}_{k}^{[r]}\right\|^{2} \mid \mathbf{y}, \hat{\boldsymbol{\lambda}}^{[m]}\right\} \\
& =E_{1}-\sum_{k=1}^{K} \frac{1}{\gamma_{k} \sigma_{w}^{2}}\left\|\hat{\mathbf{z}}_{k}^{[m]}-\eta_{k} \boldsymbol{\Lambda}_{k} \mathbf{G}_{k} \mathbf{t}_{k}^{[r]}\right\|^{2}
\end{aligned}
$$

where $E_{1}=-L Q \sum_{k=1}^{K} \log \left(\pi \gamma_{k} \sigma_{w}^{2}\right)$ is a constant that is independent of $\boldsymbol{\lambda}$. Since $\sum_{k=1}^{K} \mathbf{z}_{k}=\mathbf{y}$, it can be concluded that $\mathbf{z}_{k}$ and $\mathbf{y}$ are jointly Gaussian distributed, $\forall k$. Therefore, $\hat{\mathbf{z}}_{k}$ in (24) is given by

$$
\begin{aligned}
\hat{\mathbf{z}}_{k}^{[m]} \triangleq & \mathbb{E}\left\{\mathbf{z}_{k} \mid \mathbf{y}, \hat{\boldsymbol{\lambda}}^{[m]}\right\} \\
= & \hat{\eta}_{k}^{[m]} \hat{\boldsymbol{\Lambda}}_{k}^{[m]} \hat{\mathbf{G}}_{k}^{[m]} \mathbf{t}_{k}^{[r]} \\
& +\gamma_{k}\left(\mathbf{y}-\sum_{k=1}^{K} \hat{\eta}_{k}^{[m]} \hat{\boldsymbol{\Lambda}}_{k}^{[m]} \hat{\mathbf{G}}_{k}^{[m]} \mathbf{t}_{k}^{[r]}\right)
\end{aligned}
$$

where $\left.\hat{\boldsymbol{\Lambda}}_{k}^{[m]} \triangleq \boldsymbol{\Lambda}_{k}\right|_{\nu_{k}=\hat{\nu}_{k}^{[m]}}$ and $\left.\hat{\mathbf{G}}_{k}^{[m]} \triangleq \mathbf{G}_{k}\right|_{\tau_{k}=\hat{\tau}_{k}^{[m]}}$. b) M-Step: The estimate of $\boldsymbol{\lambda}$ at the $(m+1)$ th iteration, $\hat{\lambda}^{[m+1]}$, is determined as

$$
\begin{aligned}
\hat{\boldsymbol{\lambda}}^{[m+1]} & =\arg \max _{\boldsymbol{\lambda}} \mathbf{N}\left(\boldsymbol{\lambda} \mid \hat{\boldsymbol{\lambda}}^{[m]}\right) \\
& =\arg \min _{\boldsymbol{\lambda}} \sum_{k=1}^{K}\left\|\hat{\mathbf{z}}_{k}^{[m]}-\eta_{k} \boldsymbol{\Lambda}_{k} \mathbf{G}_{k} \mathbf{t}_{k}^{[r]}\right\|^{2} .
\end{aligned}
$$

From (26) it can be observed that the process of updating $\lambda$ can be decoupled into the processes of updating each of the $\boldsymbol{\lambda}_{k}$, for $k=\{1, \ldots, K\}$. As a result, the update-equation for calculating $\hat{\boldsymbol{\lambda}}_{k}^{[m+1]}$ can be determined as

$$
\hat{\boldsymbol{\lambda}}_{k}^{[m+1]}=\arg \min _{\boldsymbol{\lambda}_{k}}\left\|\hat{\mathbf{z}}_{k}^{[m]}-\eta_{k} \boldsymbol{\Lambda}_{k} \mathbf{G}_{k} \mathbf{t}_{k}^{[r]}\right\|^{2} .
$$

In order to further reduce the complexity associated with the $M$-step of the EM algorithm, the ECM scheme [36] is applied in this section, where the cost function in (27) is minimized with respect to to one of the parameters of interest while keeping the remaining parameters at their most recently updated values [35], [36]. In the first step using the ECM approach, $\hat{\nu}_{k}^{[m+1]}$ can be determined as

$$
\begin{aligned}
\hat{\nu}_{k}^{[m+1]}= & \left.\arg \min _{\nu_{k}}\left\|\hat{\mathbf{z}}_{k}^{[m]}-\eta_{k} \boldsymbol{\Lambda}_{k} \mathbf{G}_{k} \mathbf{t}_{k}^{[r]}\right\|^{2}\right|_{\substack{\tau_{k}=\tilde{\tau}_{k}^{[m]} \\
\eta_{k}=\hat{\eta}_{k}^{[m]}}} \\
= & \arg \max _{\nu_{k}} \sum_{i=0}^{L Q-1} \Re\left\{\left(\hat{z}_{k}^{[m]}(i)\right)^{*}\right. \\
& \left.\times \hat{\eta}_{k}^{[m]} e^{j 2 \pi i \nu_{k} / Q} b_{i}\left(\hat{\tau}_{k}^{[m]}\right)\right\}
\end{aligned}
$$

where $\hat{z}_{k}^{[m]}(i)$ is the $i$ th element of $\hat{\mathbf{z}}_{k}^{[m]}$ for $i=\{0,1, \ldots, L Q-$ $1\}$ and $b_{i}\left(\hat{\tau}_{k}^{[m]}\right)$ can be found using

$$
b_{i}\left(\tau_{k}\right)=\sum_{\ell=-L g}^{L g} t_{k}^{[r]}(\ell+\lfloor i / Q\rfloor) g\left(\bmod (i / Q) T_{s}-\ell T-\tau_{k} T\right)
$$

where $L_{g}$ is the selected pulse shaping filter lag in the TP. In order to handle the nonlinearity of (28), we can approximate the term $e^{j 2 \pi i \nu_{k} / Q}$ using Taylor series expansion to the secondorder term as

$$
\begin{aligned}
e^{j 2 \pi i \nu_{k} / Q} \approx & e^{j 2 \pi i \hat{\nu}_{k}^{[m]} / Q}+\left(\nu_{k}-\hat{\nu}_{k}^{[m]}\right)(j 2 \pi i / Q) e^{j 2 \pi i \hat{\nu}_{k}^{[m]} / Q} \\
& +\frac{1}{2}\left(\nu_{k}-\hat{\nu}_{k}^{[m]}\right)^{2}(j 2 \pi i / Q)^{2} e^{j 2 \pi i \hat{\nu}_{k}^{[m]} / Q} \cdot \quad \text { (30) }
\end{aligned}
$$

Using (30), (28) can be rewritten as in (31)

$$
\begin{aligned}
\nu_{k}^{[m+1]}= & \arg \max _{\nu_{k}} \boldsymbol{\Xi} \\
\triangleq & \sum_{i=0}^{L Q-1} \Re\left\{\left(\hat{z}_{k}^{[m]}(i)\right)^{*} \hat{\eta}_{k}^{[m]} e^{j 2 \pi i \hat{\nu}_{k}^{[m]} / Q} b_{i}\left(\hat{\tau}_{k}^{[m]}\right)\right\} \\
& -\left(\nu_{k}-\hat{\nu}_{k}^{[m]}\right) \sum_{i=0}^{L Q-1}\left(\frac{2 \pi i}{Q}\right) \\
& \times \Im\left\{\left(\hat{z}_{k}^{[m]}(i)\right)^{*} \hat{\eta}_{k}^{[m]} e^{j 2 \pi i \hat{\nu}_{k}^{[m]} / Q} b_{i}\left(\hat{\tau}_{k}^{[m]}\right)\right\} \\
& -\frac{1}{2}\left(\nu_{k}-\hat{\nu}_{k}^{[m]}\right)^{2} \sum_{i=0}^{L Q-1}\left(\frac{2 \pi i}{Q}\right)^{2} \\
& \times \Re\left\{\left(\hat{z}_{k}^{[m]}(i)\right)^{*} \hat{\eta}_{k}^{[m]} e^{j 2 \pi i \hat{\nu}_{k}^{[m]} / Q} b_{i}\left(\hat{\tau}_{k}^{[m]}\right)\right\} .
\end{aligned}
$$


Differentiating $\boldsymbol{\Xi}$ in (31) w.r.t. $\nu_{k}$ and equating the result to zero, the estimate of $\hat{\nu}_{k}$ at the $(m+1)$ th iteration is obtained as

$\hat{\nu}_{k}^{[m+1]}=\hat{\nu}_{k}^{[m]}$

$-\frac{\sum_{i=0}^{L Q-1}\left(\frac{2 \pi i}{Q}\right) \Im\left\{\left(\hat{z}_{k}^{[m]}(i)\right)^{*} \hat{\eta}_{k}^{[m]} e^{j 2 \pi i \hat{\nu}_{k}^{[m]} / Q} b_{i}\left(\hat{\tau}_{k}^{[m]}\right)\right\}}{\sum_{i=0}^{L Q-1}\left(\frac{2 \pi i}{Q}\right)^{2} \Re\left\{\left(\hat{z}_{k}^{[m]}(i)\right)^{*} \hat{\eta}_{k}^{[m]} e^{j 2 \pi i \hat{\nu}_{k}^{[m]} / Q} b_{i}\left(\hat{\tau}_{k}^{[m]}\right)\right\}}$

In the second step, by setting $\nu_{k}$ to its latest updated value, $\hat{\nu}_{k}^{[m+1]}$, the updated value of $\tau_{k}$ at the $(m+1)$ th iteration, $\hat{\tau}_{k}^{[m+1]}$, can be determined as

$$
\begin{aligned}
\hat{\tau}_{k}^{[m+1]}= & \left.\arg \min _{\tau_{k}}\left\|\hat{\mathbf{z}}_{k}^{[m]}-\eta_{k} \boldsymbol{\Lambda}_{k} \mathbf{G}_{k} \mathbf{t}_{k}^{[r]}\right\|^{2}\right|_{\substack{\nu_{k}=\hat{\nu} \\
\eta_{k}[m+1] \\
\eta_{k}=\tilde{\eta}_{k}^{[m]}}} \\
= & \arg \max _{\tau_{k}} \sum_{i=0}^{L Q-1} \Re\left\{\left(\hat{z}_{k}^{[m]}(i)\right)^{*}\right. \\
& \left.\times \hat{\eta}_{k}^{[m]} e^{j 2 \pi i \hat{\nu}_{k}^{[m+1]} / Q} b_{i}\left(\tau_{k}\right)\right\} .
\end{aligned}
$$

Taylor series expansion is again applied to linearize the maximization in (33), where the Taylor series expansion of $b_{i}\left(\tau_{k}\right)$, in (29), can be calculated as

$$
\begin{aligned}
b_{i}\left(\tau_{k}\right) \approx b_{i}\left(\hat{\tau}_{k}^{[m]}\right)+ & \left.\left(\tau_{k}-\hat{\tau}_{k}^{[m]}\right) b_{i}^{\prime}\left(\tau_{k}\right)\right|_{\tau_{k}=\hat{\tau}_{k}^{[m]}} \\
& +\left.\frac{1}{2}\left(\tau_{k}-\hat{\tau}_{k}^{[m]}\right)^{2} b_{i}^{\prime \prime}\left(\tau_{k}\right)\right|_{\tau_{k}=\hat{\tau}_{k}^{[m]}} .
\end{aligned}
$$

In (34), $b_{i}^{\prime}\left(\tau_{k}\right)$ and $b_{i}^{\prime \prime}\left(\tau_{k}\right)$ are the first and second order derivatives of the function $b_{i}\left(\tau_{k}\right)$ w.r.t. $\tau_{k}$ and are given by

$$
b_{i}^{\prime}\left(\tau_{k}\right)=\sum_{\ell=-L g}^{L g} t_{k}^{[r]}(\ell+\lfloor i / Q\rfloor) g^{\prime}\left(\bmod (i / Q) T_{s}-\ell T-\tau_{k} T\right)
$$

$b_{i}^{\prime \prime}\left(\tau_{k}\right)=\sum_{\ell=-L g}^{L g} t_{k}^{[r]}(\ell+\lfloor i / Q\rfloor) g^{\prime \prime}\left(\bmod (i / Q) T_{s}-\ell T-\tau_{k} T\right)$

where $\left.g^{\prime}(t)\right|_{\tau_{k}}=\frac{\partial}{\partial \tau_{k}} g(t)$ and $\left.g^{\prime \prime}(t)\right|_{\tau_{k}}=\frac{\partial^{2}}{\left(\partial \tau_{k}\right)^{2}} g(t)$ are the first- and second-order derivatives of transmitted pulse shaping function, $g(t)$, evaluated at $\tau=\tau_{k}$. Using forward difference approximation of derivatives given in [37], $g^{\prime}(t)$ and $g^{\prime \prime}(t)$ can be determined, respectively, as

$$
\begin{aligned}
\left.g^{\prime}(t)\right|_{\tau_{k}} & \simeq \frac{\left.g(t)\right|_{\tau_{k}+\delta}-\left.g(t)\right|_{\tau_{k}}}{\delta} \\
\left.g^{\prime \prime}(t)\right|_{\tau_{k}} & \simeq \frac{\left.g(t)\right|_{\tau_{k}+2 \delta}-\left.2 g(t)\right|_{\tau_{k}+\delta}+\left.g(t)\right|_{\tau_{k}}}{\delta^{2}}
\end{aligned}
$$

as $\delta \rightarrow 0^{+}$. Using (34), (33) can be rewritten as in (37).

$$
\begin{gathered}
\hat{\tau}_{k}^{[m+1]}=\arg \max _{\tau_{k}} \sum_{i=0}^{L Q-1} \Re\left\{\left(\hat{z}_{k}^{[m]}(i)\right)^{*} \hat{\eta}_{k}^{[m]} e^{j 2 \pi i \hat{\nu}_{k}^{[m+1]} / Q} b_{i}\left(\hat{\tau}_{k}^{[m]}\right)\right\} \\
+\left(\tau_{k}-\hat{\tau}_{k}^{[m]}\right) \sum_{i=0}^{L Q-1} \Re\left\{\left(\hat{z}_{k}^{[m]}(i)\right)^{*} \hat{\eta}_{k}^{[m]} e^{j 2 \pi i \hat{\nu}_{k}^{[m+1]} / Q} b_{i}^{\prime}\left(\hat{\tau}_{k}^{[m]}\right)\right\}
\end{gathered}
$$

$$
+\frac{1}{2}\left(\tau_{k}-\hat{\tau}_{k}^{[m]}\right)^{2} \sum_{i=0}^{L Q-1} \Re\left\{\left(\hat{z}_{k}^{[m]}(i)\right)^{*} \hat{\eta}_{k}^{[m]} e^{j 2 \pi i \hat{\nu}_{k}^{[m+1]} / Q} b_{i}^{\prime \prime}\left(\hat{\tau}_{k}^{[m]}\right)\right\} .
$$

By taking the derivative of (37) w.r.t. $\tau_{k}$ and equating the result to zero, the estimate of $\hat{\tau}_{k}$ at the $(m+1)$ th iteration is given by

$$
\begin{aligned}
& \hat{\tau}_{k}^{[m+1]}=\hat{\tau}_{k}^{[m]} \\
& \quad-\frac{\sum_{i=0}^{L Q-1} \Re\left\{\left(\hat{z}_{k}^{[m]}(i)\right)^{*} \hat{\eta}_{k}^{[m]} e^{j 2 \pi i \hat{\nu}_{k}^{[m+1]} / Q} b_{i}^{\prime}\left(\hat{\tau}_{k}^{[m]}\right)\right\}}{\sum_{i=0}^{L Q-1} \Re\left\{\left(\hat{z}_{k}^{[m]}(i)\right)^{*} \hat{\eta}_{k}^{[m]} e^{j 2 \pi i \hat{\nu}_{k}^{[m+1]} / Q} b_{i}^{\prime \prime}\left(\hat{\tau}_{k}^{[m]}\right)\right\}} .
\end{aligned}
$$

Finally, in the third step, by setting $\nu_{k}$ and $\tau_{k}$ to $\hat{\nu}_{k}^{[m+1]}$ and $\hat{\tau}_{k}^{[m+1]}$, respectively, the estimate of $\eta_{k}$, at the $(m+1)$ th iteration is calculated as

$$
\begin{aligned}
\hat{\eta}_{k}^{[m+1]}= & \left.\arg \min _{\eta_{k}}\left\|\hat{\mathbf{z}}_{k}^{[m]}-\eta_{k} \mathbf{\Lambda}_{k} \mathbf{G}_{k} \mathbf{t}_{k}^{[r]}\right\|^{2}\right|_{\substack{\nu_{k}=\hat{\nu}_{k}^{[m+1]} \\
\tau_{k}=\hat{\tau}_{k}^{[m+1]}}} \\
= & \arg \min _{\eta_{k}} \sum_{i=0}^{L Q-1} \mid \hat{z}_{k}^{[m]}(i) \\
& -\left.\eta_{k} e^{j 2 \pi i \hat{\nu}_{k}^{[m+1]} / Q} b_{i}\left(\hat{\tau}_{k}^{[m+1]}\right)\right|^{2} .
\end{aligned}
$$

By taking the derivative of (39) w.r.t. $\eta_{k}$ and setting the result to zero, $\hat{\eta}_{k}^{[m+1]}$ can be determined as

$$
\begin{aligned}
\hat{\eta}_{k}^{[m+1]}=\frac{1}{\sum_{i=0}^{L Q-1}\left|b_{i}\left(\hat{\tau}_{k}^{[m+1]}\right)\right|^{2}} & \\
& \quad \times \sum_{i=0}^{L Q-1} \frac{\hat{z}_{k}^{[m]}(i)\left(b_{i}\left(\hat{\tau}_{k}^{[m+1]}\right)\right)^{*}}{e^{j 2 \pi i \hat{\nu}_{k}^{[m+1]} / Q}} .
\end{aligned}
$$

The proposed ECM estimator for DF relaying is summarized in Table I. By reapplying the above algorithm, for $k=\{1 \ldots, K\}$, estimates of MCFOs, MTOs, and multiple channel gains for all the relays can be obtained at $\mathbb{D}$. The iterations stop when the difference between LLFs of two iterations is smaller than a threshold $\chi$, i.e.

$$
\begin{aligned}
\|\| \mathbf{y}-\sum_{k=1}^{K} \hat{\eta}_{k}^{[m+1]} \hat{\boldsymbol{\Lambda}}_{k}^{[m+1]} \hat{\mathbf{G}}_{k}^{[m+1]} \mathbf{t}_{k}^{[r]} \|^{2} \\
-\left\|\mathbf{y}-\sum_{k=1}^{K} \hat{\eta}_{k}^{[m]} \hat{\boldsymbol{\Lambda}}_{k}^{[m]} \hat{\mathbf{G}}_{k}^{[m]} \mathbf{t}_{k}^{[r]}\right\|^{2} \mid \leq \chi .
\end{aligned}
$$

3) SAGE Estimator for DF Relaying: As shown in [38], the SAGE algorithm can be applied to improve the convergence rate of the ECM approach. Using SAGE, the parameter $\boldsymbol{\lambda}$ is divided into $K$ groups denoted by $\boldsymbol{\lambda}_{k}$, for $k=\{1 \ldots, K\}$. During the estimation process each group is updated while keeping the remaining groups fixed at their latest updated values. In addition, for each group a hidden data set is selected [38]. In this case, the hidden data set denoted by $\mathbf{x}_{k}$ for $\boldsymbol{\lambda}_{k}$ is given by

$$
\mathbf{x}_{k}=\eta_{k} \boldsymbol{\Lambda}_{k} \mathbf{G}_{k} \mathbf{t}_{k}^{[r]}+\mathbf{w} .
$$


TABLE I

Proposed ECM ALGORITHM FOR DF COOPERATIVE SYSTEMS

\section{Initialization}

Obtain $\hat{\nu}_{k}^{[0]}, \hat{\tau}_{k}^{[0]}$, and $\hat{\eta}_{k}^{[0]}$ for $k=1, \ldots, K$ using alternating projection and (20) with coarser step size i.e., 0.01

ECM for DF systems

$$
\begin{aligned}
& \text { for } m=0,1, \ldots \text {. } \\
& \text { for } k=1,2, \ldots, K \\
& \hat{\mathbf{z}}_{k}^{[m]}=\hat{\eta}_{k}^{[m]} \hat{\boldsymbol{\Lambda}}_{k}^{[m]} \hat{\mathbf{G}}_{k}^{[m]} \mathbf{t}_{k}^{[r]}+\gamma_{k}\left(\mathbf{y}-\sum_{k=1}^{K} \hat{\eta}_{k}^{[m]} \hat{\boldsymbol{\Lambda}}_{k}^{[m]} \hat{\mathbf{G}}_{k}^{[m]} \mathbf{t}_{k}^{[r]}\right) \\
& \text { end } \\
& \text { for } k=1,2, \ldots, K \\
& \hat{\nu}_{k}^{[m+1]}=\hat{\nu}_{k}^{[m]}-\frac{\sum_{i=0}^{L Q-1}\left(\frac{2 \pi i}{Q}\right) \Im\left\{\left(\hat{z}_{k}^{[m]}(i)\right)^{*} \hat{\eta}_{k}^{[m]} e^{j 2 \pi i \hat{\nu}_{k}^{[m]} / Q} b_{i}\left(\hat{\tau}_{k}^{[m]}\right)\right\}}{\sum_{i=0}^{L Q-1}\left(\frac{2 \pi i}{Q}\right)^{2} \Re\left\{\left(\hat{z}_{k}^{[m]}(i)\right)^{*} \hat{\eta}_{k}^{[m]} e^{\left.j 2 \pi i \hat{\nu}_{k}^{[m]} / Q_{b_{i}\left(\hat{\tau}_{k}^{[m]}\right)}^{[m]}\right\}}\right.} \\
& \hat{\tau}_{k}^{[m+1]}=\hat{\tau}_{k}^{[m]}-\frac{\sum_{i=0}^{L Q-1} \Re\left\{\left(\hat{z}_{k}^{[m]}(i)\right)^{*} \hat{\eta}_{k}^{[m]} e^{j 2 \pi i \hat{\nu}_{k}^{[m+1]} / Q} b_{i}^{\prime}\left(\hat{\tau}_{k}^{[m]}\right)\right\}}{\sum_{i=0}^{L Q-1} \Re\left\{\left(\hat{z}_{k}^{[m]}(i)\right)^{*} \hat{\eta}_{k}^{[m]} e^{j 2 \pi i \hat{\nu}_{k}^{[m+1]} / Q} b_{i}^{\prime \prime}\left(\hat{\tau}_{k}^{[m]}\right)\right\}} \\
& \hat{\eta}_{k}^{[m+1]}=\frac{1}{\sum_{i=0}^{L Q-1}\left|b_{i}\left(\hat{\tau}_{k}^{[m+1]}\right)\right|^{2}} \sum_{i=0}^{L Q-1} \frac{\hat{z}_{k}^{[m]}(i)\left(b_{i}\left(\hat{\tau}_{k}^{[m+1]}\right)\right)^{*}}{e^{j 2 \pi i \hat{\nu}_{k}^{[m+1]} / Q}} . \\
& \hat{\nu}_{k}^{[m]}=\hat{\nu}_{k}^{[m+1]}, \hat{\tau}_{k}^{[m]}=\hat{\tau}_{k}^{[m+1]}, \hat{\eta}_{k}^{[m]}=\hat{\eta}_{k}^{[m+1]} \text {. } \\
& \text { end }
\end{aligned}
$$

The updating process for $\boldsymbol{\lambda}_{k}$ at the $m$ th iteration in the proposed SAGE estimator consists of $E$ - and $M$-Steps, which are derived using the steps outlined for the proposed ECM algorithm.

a) E-Step: While setting $\boldsymbol{\lambda}_{\ell}=\hat{\boldsymbol{\lambda}}_{\ell}^{[m]} \forall \ell \neq k$, the expectation of the LLF of the hidden data set for the parameter $\boldsymbol{\lambda}_{k}$, $\mathbf{N}\left(\boldsymbol{\lambda}_{k} \mid \hat{\boldsymbol{\lambda}}^{[m]}\right)$, is determined as

$$
\begin{aligned}
\mathbf{N}\left(\boldsymbol{\lambda}_{k} \mid \hat{\boldsymbol{\lambda}}^{[m]}\right) & \\
& \triangleq \mathbb{E}\left\{\log f\left(\mathbf{x}_{k} \mid \boldsymbol{\lambda}_{k},\left\{\hat{\boldsymbol{\lambda}}_{\ell}^{[m]}\right\}_{\ell \neq k}\right) \mid \mathbf{y}, \hat{\boldsymbol{\lambda}}^{[m]}\right\}
\end{aligned}
$$

where

$$
\begin{aligned}
f\left(\mathbf{x}_{k} \mid \boldsymbol{\lambda}_{k},\left\{\hat{\boldsymbol{\lambda}}_{\ell}^{[m]}\right\}_{\ell \neq k}\right)= & f\left(\mathbf{x}_{k} \mid \boldsymbol{\lambda}_{k}\right) \\
= & \frac{1}{\left(\pi \sigma_{w}^{2}\right)^{L Q}} \\
& \times \exp \left\{\frac{\left\|\mathbf{x}_{k}-\eta_{k} \boldsymbol{\Lambda}_{k} \mathbf{G}_{k} \mathbf{t}_{k}^{[r]}\right\|^{2}}{\sigma_{w}^{2}}\right\} .
\end{aligned}
$$

Substituting (44) into (43), we obtain

$$
\begin{aligned}
& \mathbf{N}\left(\boldsymbol{\lambda}_{k} \mid \hat{\boldsymbol{\lambda}}^{[m]}\right) \\
& =E_{2}-\frac{1}{\sigma_{w}^{2}} \mathbb{E}\left\{\left\|\mathbf{x}_{k}-\eta_{k} \boldsymbol{\Lambda}_{k} \mathbf{G}_{k} \mathbf{t}_{k}^{[r]}\right\|^{2} \mid \mathbf{y}, \hat{\boldsymbol{\lambda}}^{[m]}\right\} \\
& =E_{2}-\frac{1}{\sigma_{w}^{2}}\left\|\hat{\mathbf{x}}_{k}^{[m]}-\eta_{k} \boldsymbol{\Lambda}_{k} \mathbf{G}_{k} \mathbf{t}_{k}^{[r]}\right\|^{2}
\end{aligned}
$$

where

$$
\begin{aligned}
\hat{\mathbf{x}}_{k}^{[m]} \triangleq & \mathbb{E}\left\{\mathbf{x}_{k} \mid \mathbf{y}, \hat{\boldsymbol{\lambda}}^{[m]}\right\}=\hat{\eta}_{k}^{[m]} \hat{\boldsymbol{\Lambda}}_{k}^{[m]} \hat{\mathbf{G}}_{k}^{[m]} \mathbf{t}_{k}^{[r]} \\
& +\left(\mathbf{y}-\sum_{k=1}^{K} \hat{\eta}_{k}^{[m]} \hat{\boldsymbol{\Lambda}}_{k}^{[m]} \hat{\mathbf{G}}_{k}^{[m]} \mathbf{t}_{k}^{[r]}\right)
\end{aligned}
$$

TABLE II

Proposed SAGE Algorithm FOR DF COOPERATIVE Systems

\section{Initialization}

Obtain $\hat{\nu}_{k}^{[0]}, \hat{\tau}_{k}^{[0]}$, and $\hat{\eta}_{k}^{[0]}$ for $k=1, \ldots, K$ using alternating projection and (20) with coarser step size i.e., 0.01

SAGE for DF systems

$$
\begin{aligned}
& \text { for } m=0,1, \ldots \\
& \text { for } k=1,2, \ldots, K \\
& \hat{\mathbf{x}}_{k}^{[m]}=\mathbf{y}-\sum_{l=1, l \neq k}^{K} \hat{\eta}_{l}^{[m]} \hat{\boldsymbol{\Lambda}}_{l}^{[m]} \hat{\mathbf{G}}_{l}^{[m]} \mathbf{t}_{l}^{[r]} \\
& \hat{\nu}_{k}^{[m+1]}=\hat{\nu}_{k}^{[m]}-\frac{\sum_{i=0}^{L Q-1}\left(\frac{2 \pi i}{Q}\right) \Im\left\{\left(\hat{x}_{k}^{[m]}(i)\right)^{*} \hat{\eta}_{k}^{[m]} e^{j 2 \pi i \hat{\nu}_{k}^{[m]} / Q} b_{i}\left(\hat{\tau}_{k}^{[m]}\right)\right\}}{\sum_{i=0}^{L Q-1}\left(\frac{2 \pi i}{Q}\right)^{2} \Re\left\{\left(\hat{x}_{k}^{[m]}(i)\right)^{*} \hat{\eta}_{k}^{[m]} e^{j 2 \pi i \hat{\nu}_{k}^{[m]} / Q} b_{i}\left(\hat{\tau}_{k}^{[m]}\right)\right\}} \\
& \hat{\tau}_{k}^{[m+1]}=\hat{\tau}_{k}^{[m]}-\frac{\sum_{i=0}^{L Q-1} \Re\left\{\left(\hat{x}_{k}^{[m]}(i)\right)^{*} \hat{\eta}_{k}^{[m]} e^{j 2 \pi i \hat{\nu}_{k}^{[m+1]} / Q} b_{i}^{\prime}\left(\hat{\tau}_{k}^{[m]}\right)\right\}}{\sum_{i=0}^{L Q-1} \Re\left\{\left(\hat{x}_{k}^{[m]}(i)\right)^{*} \hat{\eta}_{k}^{[m]} e^{j 2 \pi i \hat{\nu}_{k}^{[m+1]} / Q} / b_{i}^{\prime \prime}\left(\hat{\tau}_{k}^{[m]}\right)\right\}} \\
& \hat{\eta}_{k}^{[m+1]}=\frac{1}{\sum_{i=0}^{L Q-1}\left|b_{i}\left(\hat{\tau}_{k}^{[m+1]}\right)\right|^{2}} \sum_{i=0}^{L Q-1} \frac{\hat{x}_{k}^{[m]}(i)\left(b_{i}\left(\hat{\tau}_{k}^{[m+1]}\right)\right)^{*}}{e^{j 2 \pi i \hat{\nu}_{k}^{[m+1]} / Q}} . \\
& \hat{\nu}_{k}^{[m]}=\hat{\nu}_{k}^{[m+1]}, \hat{\tau}_{k}^{[m]}=\hat{\tau}_{k}^{[m+1]}, \hat{\eta}_{k}^{[m]}=\hat{\eta}_{k}^{[m+1]} \\
& \text { end }
\end{aligned}
$$

$$
=\mathbf{y}-\sum_{\substack{\ell=1, \ell \neq k}}^{K} \hat{\eta}_{\ell}^{[m]} \hat{\boldsymbol{\Lambda}}_{\ell}^{[m]} \hat{\mathbf{G}}_{\ell}^{[m]} \mathbf{t}_{\ell}^{[r]}
$$

and $E_{2}=-(L Q) \log \left(\pi \sigma_{w}^{2}\right)$ is a constant independent of $\boldsymbol{\lambda}_{k}$

b) $M$-Step: In this step, the estimate of $\boldsymbol{\lambda}_{k}$ in the $(m+1)$ th iteration, $\hat{\boldsymbol{\lambda}}_{k}^{[m+1]}$, is determined as

$$
\begin{aligned}
\hat{\boldsymbol{\lambda}}_{k}^{[m+1]} & =\arg \max _{\boldsymbol{\lambda}_{k}} \mathbf{N}\left(\boldsymbol{\lambda}_{k} \mid \hat{\boldsymbol{\lambda}}^{[m]}\right) \\
& =\arg \min _{\boldsymbol{\lambda}_{k}}\left\|\hat{\mathbf{x}}_{k}^{[m]}-\eta_{k} \boldsymbol{\Lambda}_{k} \mathbf{G}_{k} \mathbf{t}_{k}^{[r]}\right\|^{2} .
\end{aligned}
$$

Using similar steps as the $M$-step of the ECM algorithm in Section IV-A2b, the computational complexity associated with the estimation of $\hat{\boldsymbol{\lambda}}^{[m+1]}$ can be further reduced. Subsequently, for the proposed SAGE estimator, by replacing $\hat{z}_{k}^{[m]}(i)$ with $\hat{x}_{k}^{[m]}(i),(32),(38)$, and (40) can be applied to estimate $\hat{\nu}_{k}^{[m+1]}$, $\hat{\tau}_{k}^{[m+1]}$, and $\hat{\eta}_{k}^{[m+1]}$, respectively. Table II summarizes the proposed SAGE estimator for DF relaying.

Remark 4: Even though it cannot be analytically shown that the proposed ECM and SAGE algorithms converge to a global maximum in [36, p. 1] and [38, p. 4], it is established that, in general, ECM and SAGE algorithms monotonically increase the LLF at every iteration and converge to a local maximum. Moreover, if the algorithms are initialized in a region suitably close to the global maximum, then sequence of estimates converge monotonically to the global maximum [38, p. 4]. In our simulations, initial rough estimates, $\hat{\nu}_{k}^{[0]}, \hat{\tau}_{k}^{[0]}, \hat{\eta}_{k}^{[0]}$, are obtained using alternating projection via (20) while using a coarse step size, e.g., $10^{-2}$. Finally, simulation results in Section VI investigate the performance of the proposed ECM and SAGE algorithms for different initialization step size values and illustrate that the proposed algorithms converge to the true estimates with the initialization step size of $10^{-2}$.

\section{B. Estimation Algorithms for AF Cooperative Networks}

In this section the LS, ECM, and SAGE algorithms for joint estimation of MCFOs, MTOs, and multiple channel gains in AF cooperative networks are derived. 
1) LS Estimator for AF Model: Based on the training signal model at $\mathbb{D}$ for AF relaying in (4), the LS estimate of the parameters $\boldsymbol{\alpha}, \overline{\boldsymbol{\nu}}$, and $\overline{\boldsymbol{\tau}}$ can be determined by minimizing the cost function

$$
\overline{\mathbf{J}}(\overline{\boldsymbol{\nu}}, \overline{\boldsymbol{\tau}}, \boldsymbol{\alpha})=\|\overline{\mathbf{y}}-\overline{\boldsymbol{\Omega}} \boldsymbol{\alpha}\|^{2}
$$

Given $\overline{\boldsymbol{\nu}}$ and $\overline{\boldsymbol{\tau}}$, the LS estimate of $\boldsymbol{\alpha}$ can be straightforwardly shown to be

$$
\hat{\boldsymbol{\alpha}}=\left(\overline{\mathbf{\Omega}}^{H} \overline{\mathbf{\Omega}}\right)^{-1} \overline{\mathbf{\Omega}}^{H} \overline{\mathbf{y}}
$$

By substituting (48) into (47), estimates of MCFOs and MTOs, $\hat{\overline{\boldsymbol{\nu}}}, \hat{\overline{\boldsymbol{\tau}}}$, respectively, are obtained via

$$
\hat{\overline{\boldsymbol{\nu}}}, \hat{\overline{\boldsymbol{\tau}}}=\arg \max _{\boldsymbol{\nu}, \boldsymbol{\tau}} \overline{\mathbf{y}}^{H} \overline{\boldsymbol{\Omega}}\left(\overline{\boldsymbol{\Omega}}^{H} \overline{\boldsymbol{\Omega}}\right)^{-1} \overline{\mathbf{\Omega}}^{H} \overline{\mathbf{y}} .
$$

The maximization in (49) needs to be carried out using a multidimensional exhaustive search over the set of possible timing and frequency offsets. Thus, in order to reduce the computational complexity associated with obtaining these estimates the maximization in (49) is carried out using AP, where the AP algorithm is initialized using a similar approach as that of DF relaying in Section IV-A1. In addition, the numerical investigation in Section VI indicates that the MCFOs and MTOs estimates obtained using (48) and (49) are close to the true estimates at mid-to-high SNR. Finally, the LS estimates of the channel gains $\boldsymbol{\alpha}$ can be obtained by substituting $\hat{\overline{\boldsymbol{\nu}}}$ and $\hat{\overline{\boldsymbol{\tau}}}$ into (48).

2) ECM Estimator for AF Relaying: The entries of the vector, $\overline{\boldsymbol{\theta}}$ in (8) can be rearranged to obtain a new parameter vector of interest $\bar{\lambda} \triangleq\left[\bar{\lambda}_{1}^{T}, \ldots, \bar{\lambda}_{K}^{T}\right]^{T}$, where $\bar{\lambda}_{k} \triangleq\left[\bar{\nu}_{k}, \bar{\tau}_{k}, \alpha_{k}\right]^{T}$ is a vector of three parameters corresponding to $\mathbb{R}_{k}$. Since the observed signal, $\overline{\mathbf{y}}$, in (4) is the incomplete data set in the case of AF relaying, we define the complete or hidden data set as $\overline{\mathbf{z}} \triangleq\left[\overline{\mathbf{z}}_{1}^{T}, \ldots, \overline{\mathbf{z}}_{K}^{T}\right]^{T}$, where

$$
\overline{\mathbf{z}}_{k}=\alpha_{k}\left(\overline{\boldsymbol{\Lambda}}_{k} \overline{\mathbf{G}}_{k} \mathbf{t}^{[s]}\right) \odot \overline{\mathbf{t}}_{k}^{[r]}\left(\tau_{k}^{[r d]}\right)+\mathbf{n}_{k} .
$$

In (50), $\mathbf{n}_{k} \sim \mathcal{C N}\left(0, \gamma_{k} \tilde{\sigma}_{n}^{2} \mathbf{I}_{L Q}\right)\left(\gamma_{k}\right.$ is defined below (21)) is obtained by decomposing the overall noise vector, $\mathbf{n} \triangleq \overline{\mathbf{\Psi}} \boldsymbol{\beta}+\mathbf{w}$, into $K$ components, such that $\sum_{k=1}^{K} \mathbf{n}_{k}=\mathbf{n}$. Based on the derivation in Appendix A, the covariance matrix for $\mathbf{n}$ is $\boldsymbol{\Sigma}_{\mathbf{n}}=\left(\sigma_{u}^{2} \sum_{k=1}^{K}\left|\beta_{k}\right|^{2}+\sigma_{w}^{2}\right) \mathbf{I}_{L Q}$. Given that $\beta_{k}=\zeta_{k} \eta_{k}$ where $\eta_{k}$ is the fading channel gain with variance $\sigma_{\eta_{k}}^{2}$, the covariance matrix for $\mathbf{n}$ can be modified to be $\tilde{\sigma}_{n}^{2} \mathbf{I}_{L Q}$, where $\tilde{\sigma}_{n}^{2} \triangleq \sigma_{u}^{2} \sum_{k=1}^{K} \sigma_{\eta_{k}}^{2} \zeta_{k}^{2}+\sigma_{w}^{2}$. Thus, the relationship between the complete and incomplete data sets is given by $\sum_{k=1}^{K} \overline{\mathbf{z}}_{k}=\overline{\mathbf{y}}$. For further iterative processing, let us denote $\hat{\bar{\lambda}}^{[m]} \triangleq\left[\left(\hat{\bar{\lambda}}_{1}^{[m]}\right)^{T}, \ldots,\left(\hat{\bar{\lambda}}_{K}^{[m]}\right)^{T}\right]^{T}$ as the estimated value of $\bar{\lambda}$ at the $m$ th iteration, where $\hat{\bar{\lambda}}_{k}^{[m]} \triangleq\left[\hat{\bar{\nu}}_{k}^{[m]}, \hat{\bar{\tau}}_{k}^{[m]}, \hat{\alpha}_{k}^{[m]}\right]^{T}$. Note that for AF relaying, the rough initial estimates, $\hat{\bar{\nu}}_{k}^{[0]}, \hat{\bar{\tau}}_{k}^{[0]}, \hat{\alpha}_{k}^{[0]}$, are obtained by applying AP on the proposed LS estimator in (48), and (49) with a coarse step size. The $E$-and $M$-steps of the proposed ECM estimator at the $m$ th iteration are derived in the following subsections.

a) E-Step: Using the received signal, $\overline{\mathbf{y}}$, and the current estimate $\hat{\bar{\lambda}}^{[m]}$, the expectation of the complete LLF given the parameter $\overline{\boldsymbol{\lambda}}, \overline{\mathbf{N}}\left(\overline{\boldsymbol{\lambda}} \mid \hat{\bar{\lambda}}^{[m]}\right)$, is computed as

$$
\overline{\mathbf{N}}\left(\overline{\boldsymbol{\lambda}} \mid \hat{\bar{\lambda}}^{[m]}\right) \triangleq \mathbb{E}\left\{\log f(\overline{\mathbf{z}} \mid \overline{\boldsymbol{\lambda}}) \mid \overline{\mathbf{y}}, \hat{\bar{\lambda}}^{[m]}\right\}
$$

where probability density function of $\overline{\mathbf{z}}$ as a function of $\bar{\lambda}$ is given by

$$
\begin{aligned}
f(\overline{\mathbf{z}} \mid \overline{\boldsymbol{\lambda}})= & \prod_{k=1}^{K} f\left(\overline{\mathbf{z}}_{k} \mid \overline{\boldsymbol{\lambda}}_{k}\right) \\
= & \prod_{k=1}^{K} \frac{1}{\left(\pi \gamma_{k} \tilde{\sigma}_{n}^{2}\right)^{L Q}} \\
& \times \exp \left\{\frac{\left\|\overline{\mathbf{z}}_{k}-\alpha_{k}\left(\overline{\boldsymbol{\Lambda}}_{k} \overline{\mathbf{G}}_{k} \mathbf{t}^{[s]}\right) \odot \overline{\mathbf{t}}_{k}^{[r]}\right\|^{2}}{\gamma_{k} \tilde{\sigma}_{n}^{2}}\right\} .
\end{aligned}
$$

where $\overline{\mathbf{t}}_{k}^{[r]}$ refers to $\overline{\mathbf{t}}_{k}^{[r]}\left(\hat{\bar{\tau}}_{k}^{[0]}\right)$ in Section IV-B. Substituting (52) into (51), we obtain

$$
\begin{aligned}
\overline{\mathbf{N}}\left(\overline{\boldsymbol{\lambda}} \mid \hat{\bar{\lambda}}^{[m]}\right)=E_{3} & \\
& \quad-\sum_{k=1}^{K} \frac{1}{\gamma_{k} \tilde{\sigma}_{n}^{2}}\left\|\hat{\overline{\mathbf{z}}}_{k}^{[m]}-\alpha_{k}\left(\overline{\boldsymbol{\Lambda}}_{k} \overline{\mathbf{G}}_{k} \mathbf{t}^{[s]}\right) \odot \overline{\mathbf{t}}_{k}^{[r]}\right\|^{2}
\end{aligned}
$$

where $E_{3}=-L Q \sum_{k=1}^{K} \log \left(\pi \gamma_{k} \tilde{\sigma}_{n}^{2}\right)$ is a constant independent of $\overline{\boldsymbol{\lambda}}$. Given that $\overline{\mathbf{z}}_{k}$ and $\overline{\mathbf{y}}$ are jointly Gaussian distributed, $\forall k$, we have

$$
\begin{aligned}
\hat{\overline{\mathbf{z}}}_{k}^{[m]} & \triangleq \mathbb{E}\left\{\overline{\mathbf{z}}_{k} \mid \overline{\mathbf{y}}, \hat{\overline{\boldsymbol{\lambda}}}^{[m]}\right\} \\
= & \hat{\alpha}_{k}^{[m]}\left(\hat{\overline{\boldsymbol{\Lambda}}}_{k}^{[m]} \hat{\overline{\boldsymbol{G}}}_{k}^{[m]} \mathbf{t}^{[s]}\right) \odot \overline{\mathbf{t}}_{k}^{[r]} \\
& +\gamma_{k}\left(\overline{\mathbf{y}}-\sum_{k=1}^{K} \hat{\alpha}_{k}^{[m]}\left(\hat{\overline{\boldsymbol{\Lambda}}}_{k}^{[m]} \hat{\overline{\mathbf{G}}}_{k}^{[m]} \mathbf{t}^{[s]}\right) \odot \overline{\mathbf{t}}_{k}^{[r]}\right)
\end{aligned}
$$

where $\left.\hat{\bar{\Lambda}}_{k}^{[m]} \triangleq \overline{\boldsymbol{\Lambda}}_{k}\right|_{\bar{\nu}_{k}=\hat{\bar{\nu}}_{k}^{[m]}}$ and $\left.\hat{\overline{\mathbf{G}}}_{k}^{[m]} \triangleq \overline{\mathbf{G}}_{k}\right|_{\bar{\tau}_{k}=\hat{\bar{\tau}}_{k}^{[m]}}$.

b) M-Step: The updated value of $\overline{\boldsymbol{\lambda}}, \hat{\bar{\lambda}}^{[m+1]}$, is determined as

$$
\begin{aligned}
\hat{\bar{\lambda}}^{[m+1]} & =\arg \max _{\bar{\lambda}} \overline{\mathbf{N}}\left(\bar{\lambda} \mid \hat{\bar{\lambda}}^{[m]}\right) \\
& =\arg \min _{\bar{\lambda}} \sum_{k=1}^{K}\left\|\hat{\overline{\mathbf{z}}}_{k}^{[m]}-\alpha_{k}\left(\overline{\boldsymbol{\Lambda}}_{k} \overline{\mathbf{G}}_{k} \mathbf{t}^{[s]}\right) \odot \overline{\mathbf{t}}_{k}^{[r]}\right\|^{2} .
\end{aligned}
$$

From (55) it can be straightforwardly observed that the updating process of $\bar{\lambda}$ can be decoupled into $K$ updating processes of $\bar{\lambda}_{k}$ for $k=\{1, \ldots, K\}$. Thus, the update equation to determine $\hat{\bar{\lambda}}_{k}^{[m+1]}$ is given by

$$
\hat{\bar{\lambda}}_{k}^{[m+1]}=\arg \min _{\overline{\boldsymbol{\lambda}}_{k}}\left\|\hat{\overline{\mathbf{z}}}_{k}^{[m]}-\alpha_{k}\left(\overline{\boldsymbol{\Lambda}}_{k} \overline{\mathbf{G}}_{k} \mathbf{t}^{[s]}\right) \odot \overline{\mathbf{t}}_{k}^{[r]}\right\|^{2} \text {. }
$$

Similar to DF relaying, the proposed ECM estimator for AF relaying minimizes (56) in three steps. Following the same steps as in Section IV-A2b, the updated value of $\bar{\nu}_{k}, \hat{\bar{\nu}}_{k}^{[m+1]}$, 
is obtained as shown in (57) at the bottom of the page, where $\bar{b}_{i}\left(\bar{\tau}_{k}\right)=\sum_{\ell=-L g}^{L g} t^{[s]}(\ell+\lfloor i / Q\rfloor) g\left(\bmod (i / Q) T_{s}-\ell T-\bar{\tau}_{k} T\right)$. Similarly, the updated value of $\bar{\tau}_{k}, \hat{\bar{\tau}}_{k}^{[m+1]}$, is shown by (58) at the bottom of the page, where $\bar{b}_{i}^{\prime}\left(\bar{\tau}_{k}\right)$ and $\bar{b}_{i}^{\prime \prime}\left(\bar{\tau}_{k}\right)$ can be obtained using (35), by replacing the sequence $t_{k}^{[r]}$ with $t^{[s]}$. Finally, the updated value of $\alpha_{k}, \hat{\alpha}_{k}^{[m+1]}$ is given by

$$
\begin{aligned}
\hat{\alpha}_{k}^{[m+1]}= & \frac{1}{\sum_{i=0}^{L Q-1}\left|\bar{b}_{i}\left(\hat{\bar{\tau}}_{k}^{[m+1]}\right)\right|^{2}\left|\bar{t}_{k}^{[r]}(i)\right|^{2}} \\
& \times \sum_{i=0}^{L Q-1} \frac{\hat{\bar{z}}_{k}^{[m]}(i)\left(\bar{t}_{k}^{[r]}(i)\right)^{*}\left(\bar{b}_{i}\left(\hat{\bar{\tau}}_{k}^{[m+1]}\right)\right)^{*}}{e^{j 2 \pi i \hat{\bar{\nu}}_{k}^{[m+1]} / Q} .} .
\end{aligned}
$$

The tabular form of the proposed ECM estimator for AF relaying can be obtained by modifying the initialization in Table I using (48) and (49) and by replacing $\hat{\mathbf{z}}_{k}^{[m]}, \hat{\nu}_{k}^{[m+1]}, \hat{\tau}_{k}^{[m+1]}$, and $\hat{\eta}_{k}^{[m+1]}$ in Table I by their counterparts, $\hat{\mathbf{z}}_{k}^{[m]}, \hat{\bar{\nu}}_{k}^{[m+1]}, \hat{\bar{\tau}}_{k}^{[m+1]}$, and $\hat{\alpha}_{k}^{[m+1]}$, given in (54), (57), (58), and (59), respectively. By reapplying the above process, for $k=\{1, \ldots, K\}$, estimates of the system parameters for all relays can be determined at $\mathbb{D}$. Similar to the DF case, the iterative process is terminated when the difference between the LLF of two iterations is smaller than $\chi$.

3) SAGE Estimator for AF Relaying: The parameter $\overline{\boldsymbol{\lambda}}$ is divided into $K$ groups of $\overline{\boldsymbol{\lambda}}_{k}$ and the hidden data set, $\overline{\mathbf{x}}_{k}$, for $\bar{\lambda}_{k}$ is defined as

$$
\overline{\mathbf{x}}_{k}=\alpha_{k}\left(\overline{\mathbf{\Lambda}}_{k} \overline{\mathbf{G}}_{k} \mathbf{t}^{[s]}\right) \odot \overline{\mathbf{t}}_{k}^{[r]}+\mathbf{n} .
$$

a) E-Step: While setting $\overline{\boldsymbol{\lambda}}_{\ell}=\hat{\overline{\boldsymbol{\lambda}}}_{\ell}^{[m]}, \forall \ell \neq k$, the expectation of the LLF of $\overline{\mathbf{x}}_{k}$, given $\overline{\boldsymbol{\lambda}}_{k}, \overline{\mathbf{N}}\left(\overline{\boldsymbol{\lambda}}_{k} \mid \hat{\bar{\lambda}}^{[m]}\right)$, is determined as

$$
\begin{aligned}
\overline{\mathbf{N}}\left(\overline{\boldsymbol{\lambda}}_{k} \mid \hat{\bar{\lambda}}^{[m]}\right) \\
\triangleq \mathbb{E}\left\{\log f\left(\overline{\mathbf{x}}_{k} \mid \overline{\boldsymbol{\lambda}}_{k},\left\{\hat{\overline{\boldsymbol{\lambda}}}_{\ell}^{[m]}\right\}_{\ell \neq k}\right) \mid \overline{\mathbf{y}}, \hat{\bar{\lambda}}^{[m]}\right\}
\end{aligned}
$$

where

$$
\begin{gathered}
f\left(\overline{\mathbf{x}}_{k} \mid \overline{\boldsymbol{\lambda}}_{k},\left\{\hat{\bar{\lambda}}_{\ell}^{[m]}\right\}_{\ell \neq k}\right) \\
=f\left(\overline{\mathbf{x}}_{k} \mid \overline{\boldsymbol{\lambda}}_{k}\right) \\
=\frac{1}{\left(\pi \tilde{\sigma}_{n}^{2}\right)^{L Q}}
\end{gathered}
$$

$$
\times \exp \left\{\frac{\left\|\overline{\mathbf{x}}_{k}-\alpha_{k}\left(\overline{\boldsymbol{\Lambda}}_{k} \overline{\mathbf{G}}_{k} \mathbf{t}^{[s]}\right) \odot \overline{\mathbf{t}}_{k}^{[r]}\right\|^{2}}{\tilde{\sigma}_{n}^{2}}\right\} .
$$

Substituting (62) into (61), we obtain

$$
\begin{aligned}
& \overline{\mathbf{N}}\left(\overline{\boldsymbol{\lambda}}_{k} \mid \hat{\bar{\lambda}}^{[m]}\right) \\
& \quad=E_{4}-\frac{1}{\tilde{\sigma}_{n}^{2}}\left\|\hat{\overline{\mathbf{x}}}_{k}^{[m]}-\alpha_{k}\left(\overline{\boldsymbol{\Lambda}}_{k} \overline{\mathbf{G}}_{k} \mathbf{t}^{[s]}\right) \odot \overline{\mathbf{t}}_{k}^{[r]}\right\|^{2}
\end{aligned}
$$

where

$$
\begin{aligned}
\hat{\mathbf{x}}_{k}^{[m]} & \triangleq \mathbb{E}\left\{\overline{\mathbf{x}}_{k} \mid \overline{\mathbf{y}}, \hat{\overline{\boldsymbol{\lambda}}}^{[m]}\right\} \\
& =\overline{\mathbf{y}}-\sum_{\ell=1, \ell \neq k}^{K} \hat{\alpha}_{\ell}^{[m]}\left(\hat{\overline{\mathbf{\Lambda}}}_{\ell}^{[m]} \hat{\overline{\mathbf{G}}}_{\ell}^{[m]} \mathbf{t}^{[s]}\right) \odot \overline{\mathbf{t}}_{\ell}^{[r]}
\end{aligned}
$$

and $E_{4}=-L Q \log \left(\pi \tilde{\sigma}_{n}^{2}\right)$ is a constant independent of $\overline{\boldsymbol{\lambda}}_{k}$.

b) $M$-Step: The estimate of $\overline{\boldsymbol{\lambda}}_{k}$ in the $(m+1)$ th iteration, $\hat{\bar{\lambda}}_{k}^{[m+1]}$, is determined as

$$
\begin{aligned}
& \hat{\bar{\lambda}}^{[m+1]}=\arg \max _{\overline{\boldsymbol{\lambda}}_{k}} \overline{\mathbf{N}}\left(\overline{\boldsymbol{\lambda}}_{k} \mid \hat{\overline{\boldsymbol{\lambda}}}^{[m]}\right) \\
& =\arg \min _{\overline{\boldsymbol{\lambda}}_{k}}\left\|\hat{\mathbf{\mathbf { x }}}_{k}^{[m]}-\alpha_{k}\left(\overline{\boldsymbol{\Lambda}}_{k} \overline{\mathbf{G}}_{k} \mathbf{t}^{[s]}\right) \odot \overline{\mathbf{t}}_{k}^{[r]}\right\|^{2}
\end{aligned}
$$

For the proposed SAGE algorithm, by replacing $\hat{\bar{z}}_{k}^{[m]}(i)$ with $\hat{\bar{x}}_{k}^{[m]}(i),(57),(58)$, and (59) can be used to estimate $\hat{\hat{\nu}}_{k}^{[m+1]}$, $\hat{\bar{\tau}}_{k}^{m+1]}$, and $\hat{\alpha}_{k}^{[m+1]}$, respectively. The tabular form of the proposed SAGE estimator for AF relaying can be obtained by modifying the initialization in Table II, using (48) and (49), and by replacing $\hat{\mathbf{x}}_{k}^{[m]}, \hat{\nu}_{k}^{[m+1]}, \hat{\tau}_{k}^{[m+1]}$, and $\hat{\eta}_{k}^{[m+1]}$ by their counterparts, $\hat{\mathbf{x}}_{k}^{[m]}, \hat{\bar{\nu}}_{k}^{[m+1]}, \hat{\bar{\tau}}_{k}^{[m+1]}$, and $\hat{\alpha}_{k}^{[m+1]}$, given in (64), (57), (58), and (59), respectively. ${ }^{5}$

\section{Complexity Analysis of Proposed Estimators}

Throughout this paper computational complexity is defined as the number of additions plus multiplications. In this subsection the computational complexity of ML estimation in [17] and the proposed ECM and SAGE algorithms for DF relaying are analyzed. In order to avoid repetition, the case of AF relaying has been omitted, since in the AF scenario the computational complexity of the proposed estimators can be determined by using the number of iterations required by each algorithm and by

${ }^{5}$ Note that Remark 4 also holds for AF relaying.

$$
\hat{\bar{\nu}}_{k}^{[m+1]}=\hat{\bar{\nu}}_{k}^{[m]}-\frac{\sum_{i=0}^{L Q-1}\left(\frac{2 \pi i}{Q}\right) \Im\left\{\left(\hat{\bar{z}}_{k}^{[m]}(i)\right)^{*} \hat{\alpha}_{k}^{[m]} e^{j 2 \pi i \hat{\nu}_{k}^{[m]} / Q} \bar{t}_{k}^{[r]}(i) \bar{b}_{i}\left(\hat{\bar{\tau}}_{k}^{[m]}\right)\right\}}{\sum_{i=0}^{L Q-1}\left(\frac{2 \pi i}{Q}\right)^{2} \Re\left\{\left(\hat{\bar{z}}_{k}^{[m]}(i)\right)^{*} \hat{\alpha}_{k}^{[m]} e^{j 2 \pi i \hat{\bar{\nu}}_{k}^{[m]} / Q} \bar{t}_{k}^{[r]}(i) \bar{b}_{i}\left(\hat{\bar{\tau}}_{k}^{[m]}\right)\right\}}
$$

$$
\hat{\bar{\tau}}_{k}^{[m+1]}=\hat{\bar{\tau}}_{k}^{[m]}-\frac{\sum_{i=0}^{L Q-1} \Re\left\{\left(\hat{\bar{z}}_{k}^{[m]}(i)\right)^{*} \hat{\alpha}_{k}^{[m]} e^{j 2 \pi i \hat{\bar{\nu}}_{k}^{[m+1]} / Q} \bar{t}_{k}^{[r]}(i) \bar{b}_{i}^{\prime}\left(\hat{\bar{\tau}}_{k}^{[m]}\right)\right\}}{\sum_{i=0}^{L Q-1} \Re\left\{\left(\hat{\bar{z}}_{k}^{[m]}(i)\right)^{*} \hat{\alpha}_{k}^{[m]} e^{j 2 \pi i \hat{\bar{\nu}}_{k}^{[m+1]} / Q} \bar{t}_{k}^{[r]}(i) \bar{b}_{i}^{\prime \prime}\left(\hat{\bar{\tau}}_{k}^{[m]}\right)\right\}}
$$


adding the additional multiplications required due to the factor $\bar{t}_{k}^{[r]}(i)$ in (57), (58), and (59). The computational complexity of the ML algorithm, denoted by $C_{\mathrm{ML}}$ is calculated as

$$
\begin{aligned}
C_{\mathrm{ML}} & =\underbrace{2 K^{2} L Q+K^{3}+K L Q}_{\boldsymbol{\nu}, \boldsymbol{T} \text { in }(20)} \\
+ & \underbrace{(\mathcal{N} 2 K / \kappa)\left[\left(2 K^{2}+1\right) L Q+(1+K)(L Q)^{2}+K^{3}\right]}_{\boldsymbol{\eta} \text { in }(20)}
\end{aligned}
$$

where $\mathcal{N}$ denotes the number of alternating projection cycles used [22], and $\kappa$ denotes the step size in the ML search in (20). The computational complexity of the proposed ECM algorithm, denoted by $C_{\mathrm{ECM}}$ is calculated as

$$
\begin{aligned}
C_{\mathrm{ECM}} & \\
= & C_{\mathrm{I}}+K \varsigma[\underbrace{12 L Q+4 L_{g} L Q+2}_{(32)} \\
& +\underbrace{10 L Q+8 L_{g} L Q+2}_{(38)}+\underbrace{6 L Q+4 L_{g} L Q+1}_{(25)} \\
& +\underbrace{(K+2) L Q+(K+1) L^{2} Q+(K+1)(L Q)^{2}+1}_{(40)} \\
& +\underbrace{2 Q\left(2 L_{g}+1\right)+2}_{(36)}]
\end{aligned}
$$

where $C_{\mathrm{I}}$ is the computational cost associated with determining the initial rough estimates given by $C_{I}=\left.C_{\mathrm{ML}}\right|_{\kappa=\mu}, \mu$ is the coarse step size used to calculate the initial estimates for the proposed ECM algorithm, and $\varsigma$ denotes the average total number of iterations required. The value of $\varsigma$ for the ECM and SAGE algorithms have been determined through numerical simulations in Section VI as illustrated in Fig. 10, e.g., in the case of DF relaying $\varsigma=29$ and 16 for ECM and SAGE algorithms, respectively, with SNR $=15$ and $K=2$ relays. Similarly, the computational complexity of the proposed SAGE algorithm, denoted by $C_{\mathrm{SAGE}}$, is calculated as

$$
\begin{aligned}
C_{\mathrm{SAGE}}= & C_{\mathrm{I}}+K \varsigma[\underbrace{12 L Q+4 L_{g} L Q+2}_{\hat{\nu}_{k}^{[m+1]} \text { in Table II }} \\
& +\underbrace{10 L Q+8 L_{g} L Q+2}_{(45)} \\
& +\underbrace{6 L Q+4 L_{g} L Q+1}_{\hat{\tau}_{k}^{[m+1]} \text { in TableII }} \\
& +\underbrace{K L Q+(K-1) L^{2} Q+(K-1)(L Q)^{2}}_{(36)} \\
& +\underbrace{K L\left(2 L_{g}+1\right)+2}_{\hat{\eta}_{k}^{[m+1]} \text { in TableII }}] .
\end{aligned}
$$

Based on (66), (67) and (68), the following remarks are in order.

Remark 5: In order to reach the CRLB for the estimation of MCFOs, MTOs, and channel gains (see Figs. 6 and 7 in
TABLE III

CPU PROCESSING TIME FOR ML, ECM AND SAGE WITH 4 RELAYS DF SYSTEM AT SNR $=20 \mathrm{~dB}$ USING INTEL CORE 2 QUAD $2.66 \mathrm{GHz}$ PROCESSOR

\begin{tabular}{|c|c|}
\hline Implemented Algorithm & CPU Processing Time (minutes) \\
\hline \hline ML [18] & 179.725 \\
\hline ECM & 0.2698 \\
\hline SAGE & 0.1945 \\
\hline
\end{tabular}

Section VI), the step size, $\kappa$ for the ML in [17] and the proposed LS estimator needs to be very small, e.g., $\kappa=10^{-5}$. This, in turn, significantly increases the computational complexity of these estimators given that the maximizations in (20) and (49) for DF and AF relaying, respectively, need to be carried out over a significantly larger set of possible values. However, a step size of say, $\mu=10^{-2}$, suffices to obtain rough initial estimates for the proposed ECM and SAGE algorithms.

Remark 6: In order to quantitatively compare the computational complexity of the ML in [17] and the proposed ECM and SAGE estimators for DF relaying, we have evaluated $C_{\mathrm{ML}}$, $C_{\mathrm{ECM}}$, and $C_{\mathrm{SAGE}}$ in (66), (67) and (68), respectively, for $K=4$ relays at an SNR of $20 \mathrm{~dB}$. It is observed that even by considering the complexity associated to the initialization step of the proposed ECM and SAGE estimators, these schemes are 772 and 946 times more computationally efficient than the ML estimator in [17] carried out using AP, respectively. The computational complexity of the proposed algorithms is also evaluated using CPU execution time [39]. For the case of DF relaying networks, Table III depicts the execution times for the ML estimator in [17], and for the proposed ECM and SAGE estimators with SNR $=20 \mathrm{~dB}$ and $K=4$ relays when an Intel Core 2 Quad 2.66 $\mathrm{GHz}$ processor processor with $4 \mathrm{~GB}$ byte of RAM is used. It can be observed from Table III that compared to the ML estimator in [17] the proposed ECM and SAGE estimators are capable of estimating the overall network's synchronization parameters and channel gains approximately 666 and 924 times more quickly.

Remark 7: The proposed ECM and SAGE algorithms need to apply the ML and LS estimators for initialization only once at system start-up. Afterwards, the estimates of previously transmitted frames may be used to update the new estimates since timing and carrier frequency offsets do not rapidly change from frame to frame. This is due to the fact that oscillator properties are mainly affected by temperature and other physical phenomena that do not rapidly fluctuate with time [40].

\section{ML DECODING}

In order to decode the received signal at $\mathbb{D}$ in the presence of multiple impairments, an ML decoder for both $\mathrm{DF}$ and $\mathrm{AF}$ multi-relay cooperative systems is proposed.

\section{A. Decoding in DF-Relaying Networks}

During the DTP, the decoder at $\mathbb{D}$ evaluates the metric, $d_{p}(i)$, according to 6

$$
\begin{aligned}
& d_{p}(i)=\sum_{k=1}^{K} \hat{\eta}_{k} e^{j 2 \pi i \hat{\nu}_{k} / Q} \sum_{\ell=-D_{g}}^{D_{g}} c_{p}(\ell, i) \\
& g\left(\lfloor i / Q\rfloor T_{s}-\ell T-\hat{\tau}_{k} T\right), \\
& p=1, \ldots, M^{D_{g}+1}
\end{aligned}
$$

${ }^{6}$ Subscript $p$ refers to the $p$ th permutation, for $p=1, \ldots, M^{D_{g}+1}$. 
TABLE IV

THE PROPOSED ML DECODER FOR DF RELAYING

$$
\begin{aligned}
& \text { for } i=0,1, \ldots, L Q-1 \\
& d_{p}(i)=\sum_{k=1}^{K} \hat{\eta}_{k} e^{j \pi i \hat{\nu}_{k} / Q} \sum_{\ell=-D_{g}}^{D_{g}} c_{p}(\ell, i) g\left(\lfloor i / Q\rfloor T_{s}-\ell T-\hat{\tau}_{k} T\right) \\
& e_{p}(i)=\left|y(i)-d_{p}(i)\right|^{2} \\
& \text { if }\left(\bmod \left(\frac{i+1}{Q}\right)==0\right) \\
& \quad \epsilon_{p}=\sum_{q=0}^{Q-1} e_{p}(i-q) \\
& p_{\text {sel }}=\arg \min _{p} \epsilon_{p} \\
& \hat{s}\left(\frac{i+1}{Q}-1\right)=a_{p_{\text {sel }}}(0) \\
& \text { end } \\
& \text { end }
\end{aligned}
$$

where $\mathbf{c}_{p}(i) \triangleq\left[c_{p}\left(-D_{g}, i\right), \ldots, c_{p}\left(D_{g}, i\right)\right]^{T}=[\hat{s}(\lfloor i / Q\rfloor-$ $\left.\left.D_{g}\right), \ldots, \hat{s}(\lfloor i / Q\rfloor-1), a_{p}(0), \ldots, a_{p}\left(D_{g}\right)\right]^{T}$ is a $\left(2 D_{g}+1\right) \times 1$ vector and $D_{g}$ is a constant pulse shaping filter lag during the DTP. Note that $\mathbf{a}_{p} \triangleq\left[a_{p}(0), \ldots, a_{p}\left(D_{g}\right)\right]^{T}$ is a $\left(D_{g}+1\right) \times$ 1 vector of the $p$ th permutation of the $M$ symbols within the constellation. Next, the error between the metric, $d_{p}(i)$, and the received signal, $y(i)$ at $\mathbb{D}$ is determined as

$$
e_{p}(i)=\left|y(i)-d_{p}(i)\right|^{2}, \quad p=1, \ldots, M^{D_{g}+1} .
$$

Thereafter, the summation of the errors, $e_{p}(i)$, in (70) that correspond to the $n$th symbol, $\epsilon_{p}(n)$, is given by

$$
\epsilon_{p}(n)=\sum_{q=0}^{Q-1} e_{p}(n Q+q), \quad p=1, \ldots, M^{D_{g}+1} .
$$

The permutation that results in the smallest error is denoted by $p_{\text {sel }}$, and is selected as

$$
p_{\text {sel }}=\arg \min _{p} \epsilon_{p}(n), \quad p=1, \ldots, M^{D_{g}+1} .
$$

Finally, using $p_{\text {sel }}$ the $n$th received symbol is decoded as $\hat{s}(n)=$ $a_{p_{\text {sel }}}(0)$, where $a_{p_{\text {sel }}}(0)$ is the zeroth element of the vector $\mathbf{a}_{p_{\text {sel }}}$ corresponding to $p_{\text {sel }}$. The proposed ML decoder for DF relaying is summarized in Table IV.

\section{B. Decoding in AF-Relaying Networks}

Similar to the DF case, the estimates $\hat{\overline{\boldsymbol{\tau}}}, \hat{\overline{\boldsymbol{\nu}}}$ and $\hat{\boldsymbol{\alpha}}$ are used to decode the received signal. The decoder at $\mathbb{D}$ in the AF system evaluates the metric

$$
\begin{aligned}
\bar{d}_{p}(i)=\sum_{k=1}^{K} \hat{\alpha}_{k} e^{j 2 \pi i \hat{\bar{\nu}}_{k} / Q} \\
\quad \times \sum_{\ell=-D_{g}}^{D_{g}} c_{p}(\ell, i) g\left(\lfloor i / Q\rfloor T_{s}-\ell T-\hat{\bar{\tau}}_{k} T\right) .
\end{aligned}
$$

Using the metric in (73) and the same steps as outlined in Section V-A, the $n$th source symbol, $\hat{s}(n)$, can be decoded. The tabular form of the ML decoder for AF relaying is given by replacing $d_{p}(i)$ with $\bar{d}_{p}(i)$ in Table IV.

Remark 8: The ML decoder outlined above is derived to showcase that the estimates obtained during the TP using the proposed estimators can be applied to effectively decode the received signal in multi-relay cooperative networks. Since, it is a well-known that the complexity of ML decoding increases exponentially with constellation size, the design of more com- putationally efficient decoders for cooperative networks in the presence of MCFOs, MTOs, and unknown channel gains could be investigated in future work ${ }^{7}$.

\section{Simulation ReSUlts}

In this section, we present simulation results to evaluate the performance of our estimators. We use quadrature phase-shift keying (QPSK) modulation. Without loss of generality, we assume $\sigma_{u}^{2}=\sigma_{w}^{2}=1 / \mathrm{SNR}$. The propagation loss is modeled as $\left(d / d_{0}\right)^{-m}$, where $d$ is the distance between transmitter and receiver, $d_{0}$ is the reference distance, and $m$ is the path loss exponent [27]. The following simulations are based on TS length, $L=64, \sigma_{\rho}^{2}=\sigma_{\eta}^{2}=1, d_{0}=1 \mathrm{~km}$, and $m=2.7$, which corresponds to urban area cellular networks. The timing offsets at $\mathbb{D}, \boldsymbol{\tau}^{[r d]}$ are assumed to be uniformly distributed over the range $(-0.5,0.5)$. Based on the methodology in [6], [31], the timing offset estimation errors from $\mathbb{S}-\mathbb{R}, \epsilon_{k}^{[s r]}$, is assumed to follow a Gaussian distribution, i.e., $\epsilon_{k}^{[s r]} \sim \mathcal{N}\left(0, \sigma_{\tau}^{2}\right)$, where $\sigma_{\tau}^{2}$ is set to the lower bound on the variance of timing offset estimation error in point-to-point systems [27, p. 328]. Carrier frequency offsets for $\mathrm{DF}$ relaying at $\mathbb{D}, \boldsymbol{\nu}^{[r d]}$, are uniformly distributed in the full acquisition range $(-0.5,0.5)$. For AF relaying, since carrier frequency offsets from source to relays, $\boldsymbol{\nu}^{[s r]}$, are carried over to the destination, $\boldsymbol{\nu}^{[s r]}$ and $\boldsymbol{\nu}^{[r d]}$ have the range $(-0.25,0.25)$ in order to limit the total frequency offset from source to destination $\nu^{[s d]}$ to the range $(-0.5,0.5)$, i.e., full acquisition. Distinct phase shift keying training sequences are generated at $\mathbb{S}$ and all relays similar to [15]. $d^{[s r]}$ and $d^{[r d]}$ are used to denote the $\mathbb{S}-\mathbb{R}$ and $\mathbb{R}-\mathbb{D}$ distances, respectively. Finally, the mean-square error (MSE) performance of various estimators and the bit error rate (BER) performance of the overall multi-relay cooperative network is detailed in the following subsections.

\section{A. Estimator Performance}

Specific channels are used for the following simulations, i.e., $\boldsymbol{\rho}=[.279-.9603 j, .8837+.4681 j,-.343+.732 i,-.734-$ $.451 i]^{T}$ and $\boldsymbol{\eta}=[.7820+.6233 j, .9474-.3203 j,-.2413+$ $.724 i, .5141-.893 i]^{T}$ similar to [7]-[9]. Unless otherwise specified, $K=4$ relays, $Q=2$ in the TP, and $d^{[s r]}=d^{[r d]}=1$ $\mathrm{km}$ are used. The remaining parameters are set as $\chi=0.001$, $\delta=0.0001, \kappa=10^{-5}, \mathcal{N}=2$ and 3 for DF and AF relaying, respectively, $\mu=10^{-2}$, and $L_{g}=10$. Finally, the MSE for the estimation of a parameter say, frequency offset, $\boldsymbol{\nu}$, is defined as the average MSE over all the simulations runs, i.e., $\operatorname{MSE}(\boldsymbol{\nu})=\sum_{m=1}^{10^{4}} \sum_{k=1}^{K}\left(\hat{\nu}_{k}^{[m]}-\nu_{k}^{[m]}\right)^{2} / 10^{4}$.

Figs. 6-7(a), (b), and (c) show the CRLB and MSE for the estimation of MTOs, MCFOs, and channel gains for DF and AF relaying, respectively. It is shown that the MSEs of the ML and proposed LS estimators for both DF and AF relaying are close to their CRLBs at mid-to-high SNRs. In comparison, the proposed ECM and SAGE estimators are close to the CRLB at mid-SNR values but exhibit some small performance degradation w.r.t. to the CRLB when estimating MCFOs, MTOs, and channel gains at high SNR. In addition, Figs. 6(a) and 7(a) indicate that while estimating MTOs at high SNR, the MSEs of

\footnotetext{
${ }^{7}$ In order to reduce complexity, the knowledge of channel gains, frequency offsets, and timing offsets can be used with a zero-forcing equalizer to decode the received signal at the destination.
} 


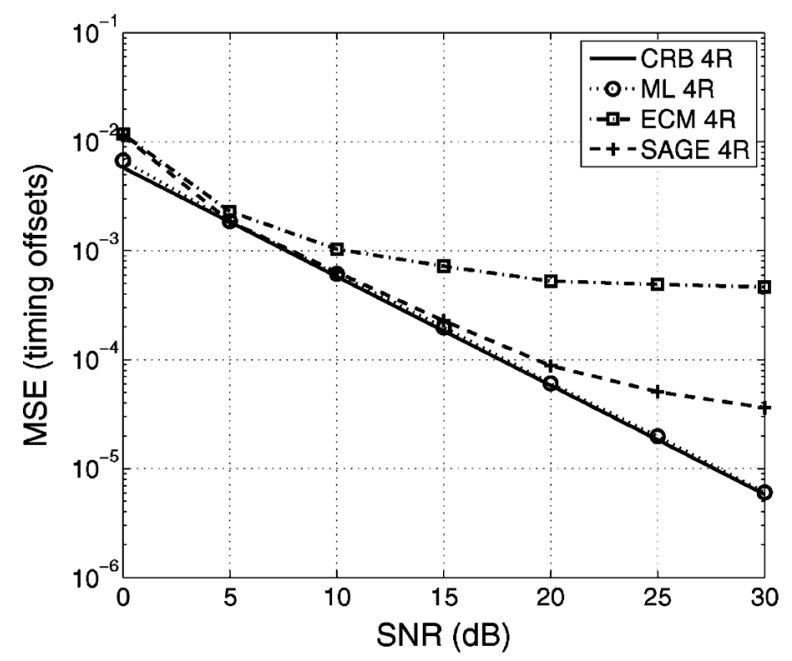

(a)

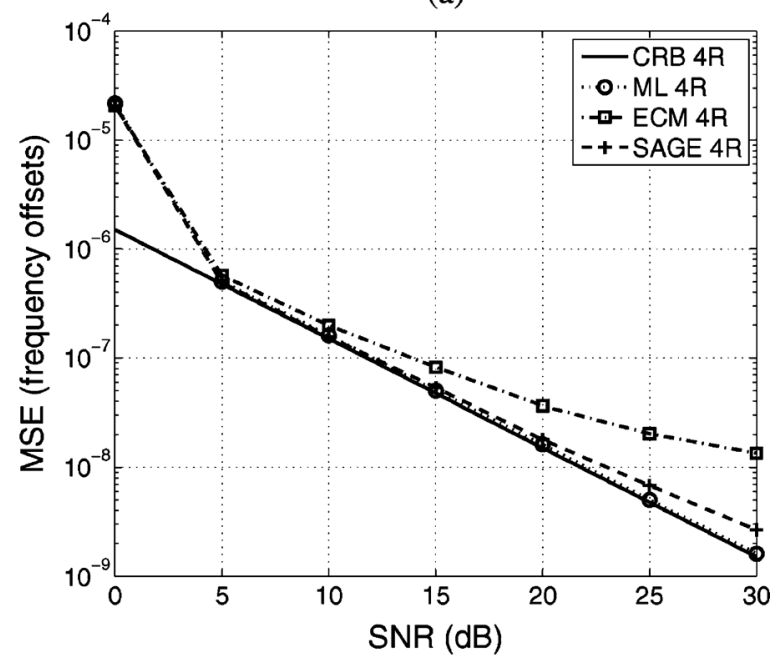

(b)

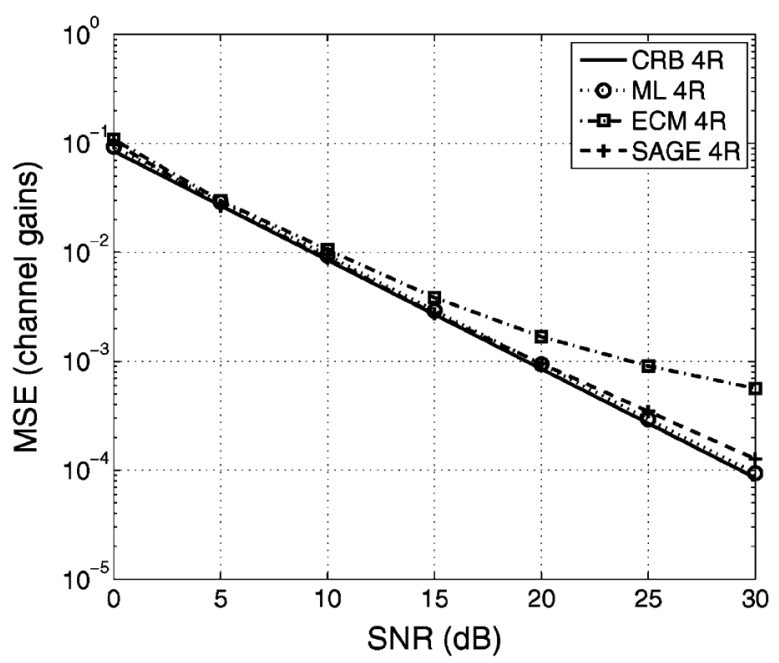

(c)

Fig. 6. MSE and CRLBs of (a) MTOs, (b) MCFOs, and (c) channel coefficients estimation as a function of SNR (in decibels) for DF relaying.

the proposed ECM and SAGE estimators exhibit an error floor. This error floor is caused by the Taylor series approximations in (30) and (34) as well as the approximation in (36), which

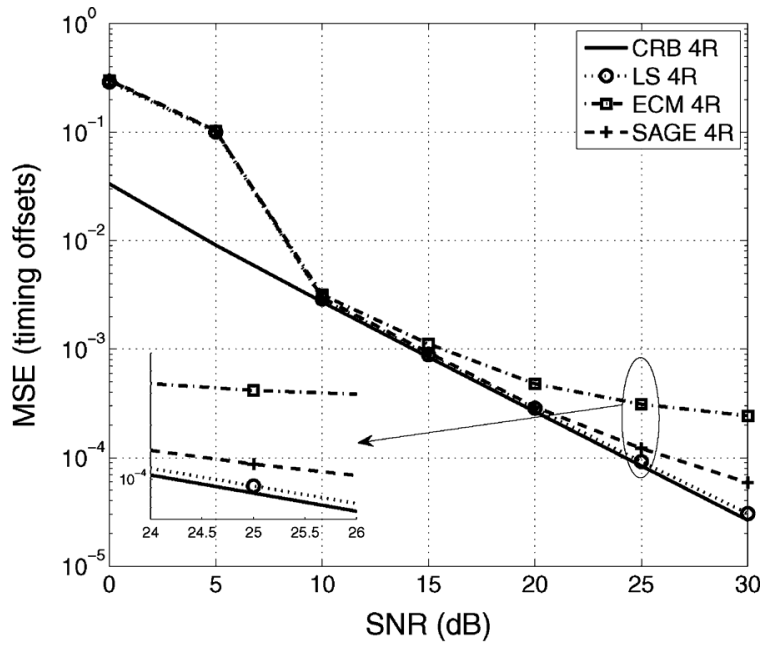

(a)

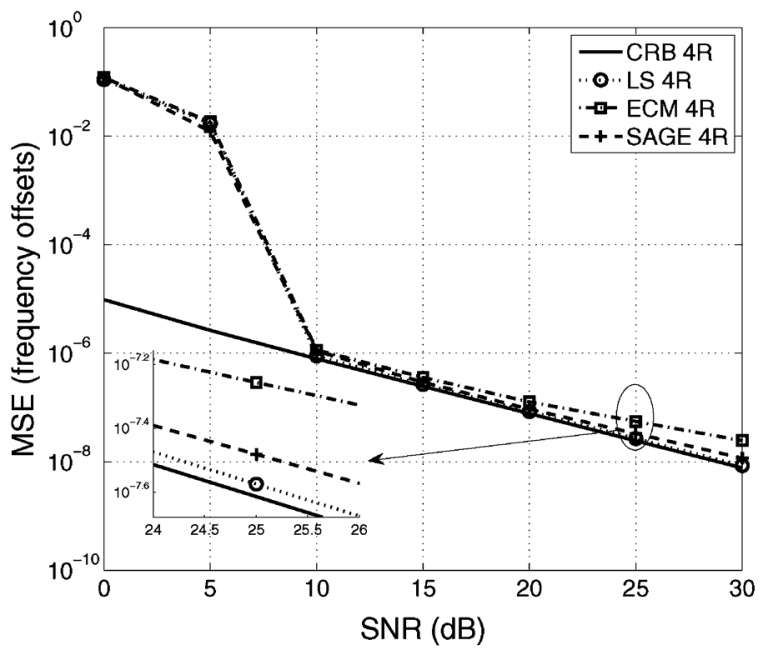

(b)

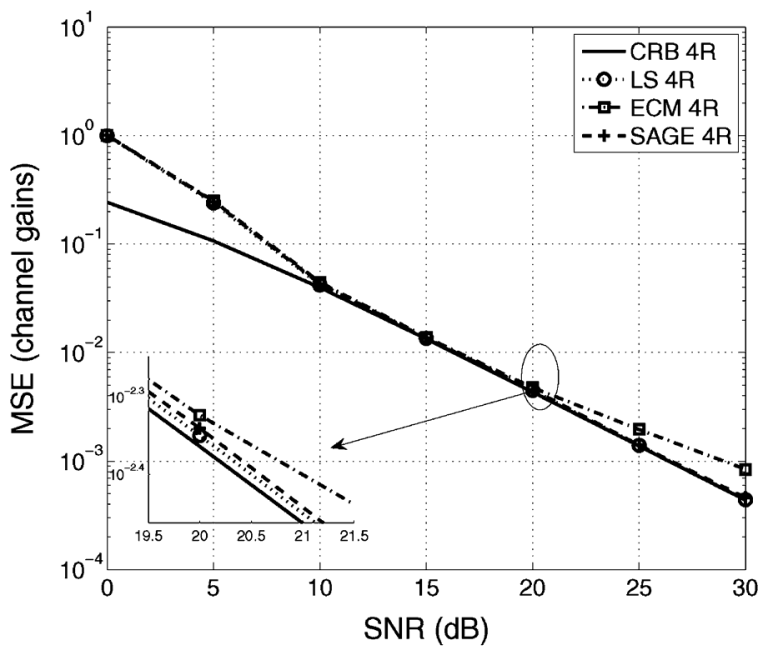

(c)

Fig. 7. MSE and CRLBs of (a) MTOs, (b) MCFOs, and (c) channel coefficients estimation as a function of SNR (in decibels) for AF system.

are used to linearize the LLF under consideration. However, as shown in Section IV-C, compared to the ML and proposed LS schemes, the proposed ECM and SAGE estimators significantly 


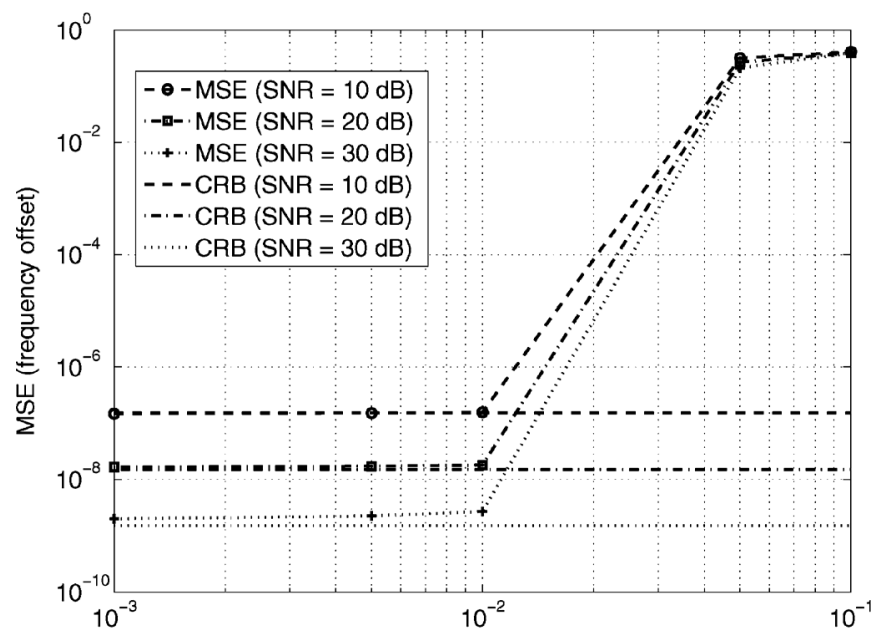

( $\mu$ ) step size for initialization of SAGE algorithm

Fig. 8. MSE of frequency offset estimation versus initialization of SAGE algorithm with different values of coarse step size, $\mu$, in DF cooperative networks.

reduce the computational complexity associated with estimating impairments in cooperative networks. Moreover, at low SNR for AF relaying, the proposed LS, ECM, and SAGE estimators demonstrate poor performance due to the considerable timing offset estimation error from source to relays and the noise at the relays which is amplified and forwarded to the destination. Finally, Figs. 6 and 7 show that the proposed SAGE estimator outperforms the ECM algorithm for all SNR values.

Fig. 8 presents the impact of the initialization of the SAGE algorithm on the estimator's performance for different step size values, $\mu$. It is shown that decreasing the step size from $\mu=$ $10^{-2}$ results in diminishing returns in frequency offset estimation accuracy since the MSE of the proposed SAGE estimator for $\mu=10^{-2}$ is already close to the CRLB. Thus, it can be concluded that for $\mu=10^{-2}$ the proposed SAGE estimator is initialized in the region of a local maximum, which turns out to be the global maximum and converges to the true estimates. Consequently, in all the simulations in this section the step size, $\mu=10^{-2}$, is used to initialize the proposed ECM and SAGE estimators ${ }^{8}$. Note that similar results to that of Fig. 8 are observed in the case of AF relaying cooperative networks and are omitted from the paper to avoid repetition.

Fig. 9 compares frequency offset estimation MSE of the proposed SAGE estimator against the MSE of the initial estimates. This result further shows that obtaining frequency offsets using the proposed SAGE algorithm significantly improves estimation accuracy. In addition, unlike the ML and LS estimators, this improvement in estimation accuracy is achieved without performing an exhaustive search over a large set of possible frequency offset values with small step size values, e.g., $10^{-5}$.

Fig. 10 shows the average number of iterations required by the proposed ECM and SAGE algorithms to converge in DF-relaying networks. It can be observed that at an SNR of $20 \mathrm{~dB}$, the average number of iterations required by the SAGE estimator is 2 and 3.4 times fewer than that of ECM algorithm for networks with 2 and 4 relays, respectively. Note that similar results are obtained for the case of AF relaying.

${ }^{8}$ Note that the ML estimator in [17] requires an exhaustive search with very small step size values, e.g., $10^{-5}$, to reach the CRLB as explained in Remark 5 .

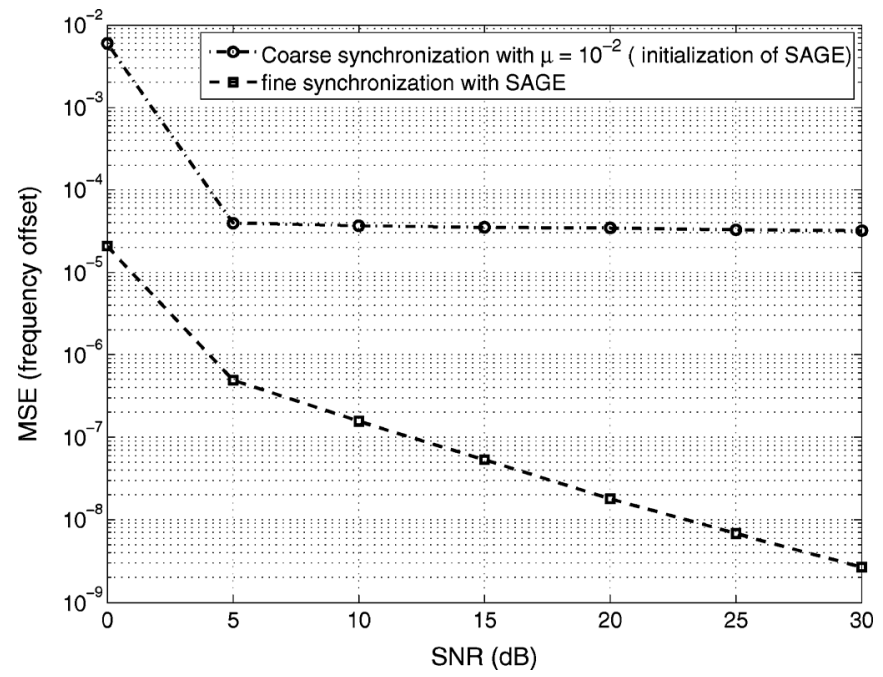

Fig. 9. MSE of frequency offset estimation with coarse initialization $(\mu=$ $\left.10^{-2}\right)$ and fine estimation using SAGE algorithm in DF cooperative networks.

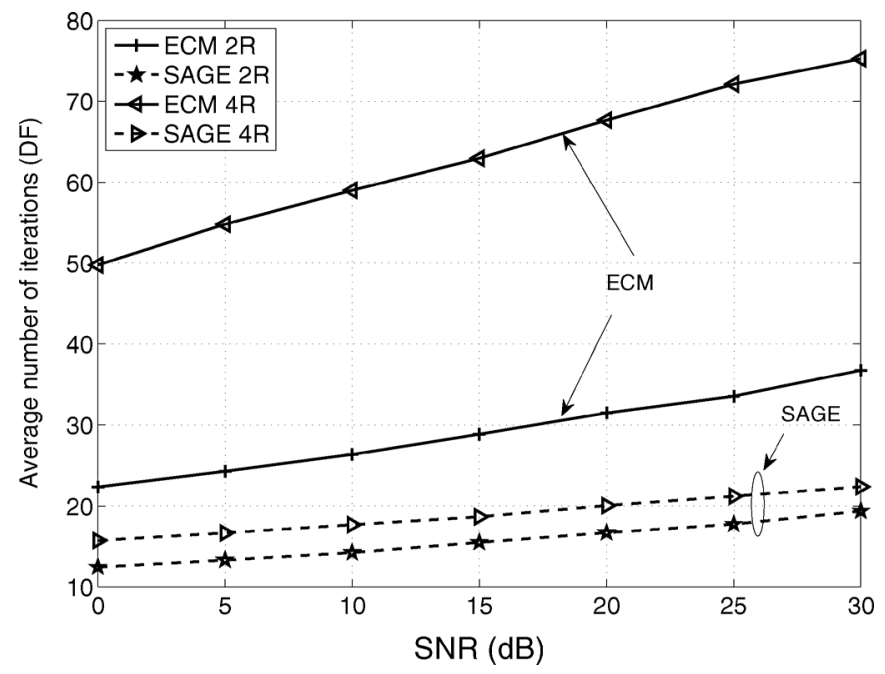

Fig. 10. Average number of iterations for ECM and SAGE in DF cooperative networks for $\mathrm{K}=2$ and 4 relays.

Fig. 11 shows the CRLB for frequency and timing offset estimation for AF relaying when relays are located at different physical locations: estimation performance slightly improves by moving the relays closer to $\mathbb{D}$, i.e., $d^{[s r]}=1.3 \mathrm{~km}$ and $d^{[r d]}=0.7 \mathrm{~km}$, due to lesser propagation loss from relays to $\mathbb{D}$. However, the performance degrades by moving the relays closer to $\mathbb{S}$, i.e., $d^{[s r]}=0.7 \mathrm{~km}$ and $d^{[r d]}=1.3 \mathrm{~km}$, due to larger propagation loss from the relays to $\mathbb{D}$. Note that the improvement in the estimation performance is lesser, while moving the relays closer to $\mathbb{D}$, due to the additional amplification of noise at $\mathbb{D}$.

\section{B. Cooperative Performance}

The channel gains from source to relays and from relays to destination are modeled as independent and identically distributed (i.i.d) complex Gaussian random variables with $\mathcal{C N}(0,1)$. We use $Q=4$ for the DTP and $D_{g}=4$ in (69) and (73) for the proposed ML decoder. Cooperative communication networks with two relays are considered, where the relays are distributed throughout the network. Fixed gain relaying is applied for AF relaying as shown in Section II-A1. We assume 


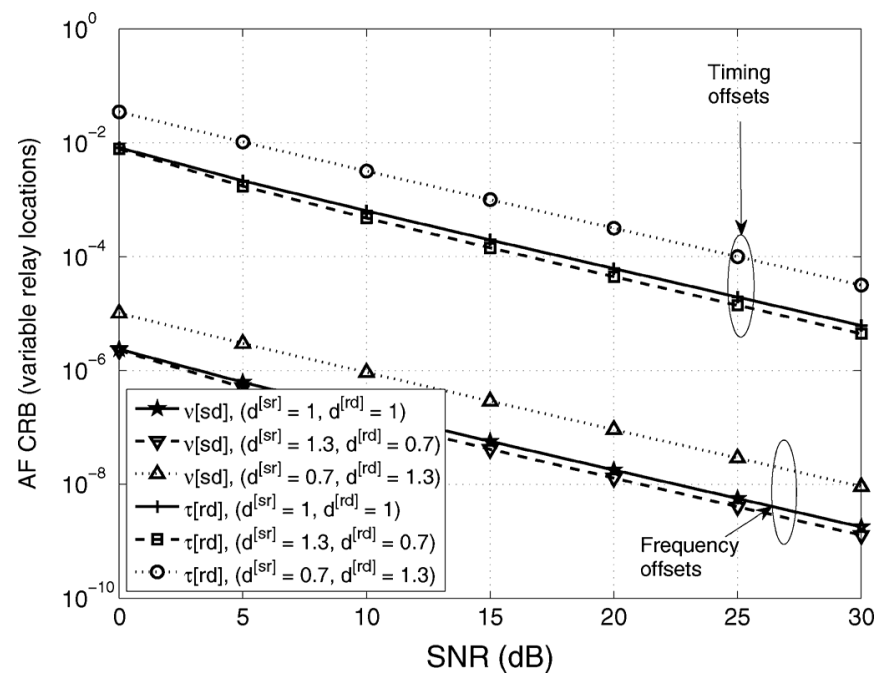

Fig. 11. CRLB for MCFOs and MTOs with AF relays at different locations.

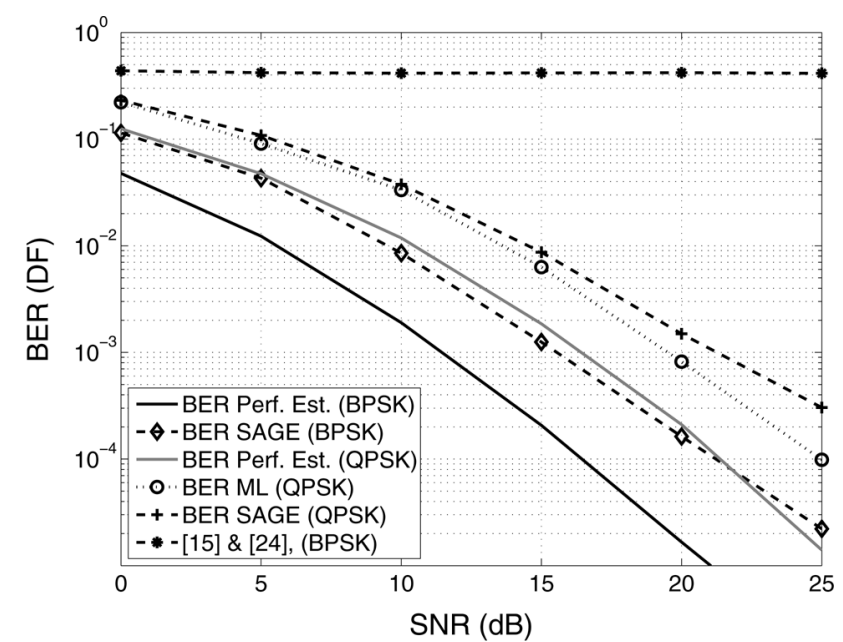

(a)

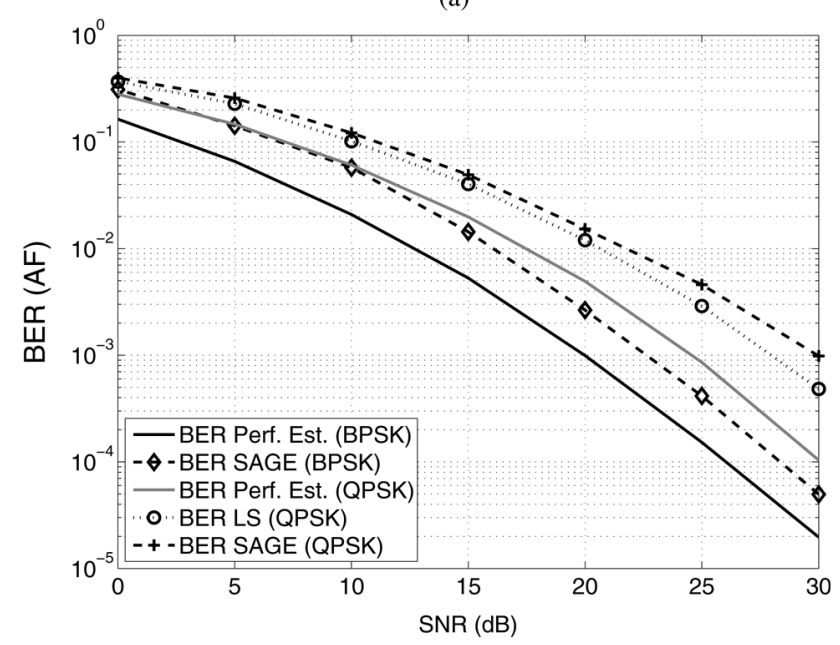

(b)

Fig. 12. (a) BER performance for a DF cooperative system with $K=2$. (b) $\mathrm{BER}$ performance for an $\mathrm{AF}$ cooperative system with $K=2$.

a training sequence length of 80 symbols and a frame length of $L=450$ symbols.

Fig. 12(a) and (b) show the BER performance with two relays for an uncoded DF and AF cooperative networks for binary phase-shift keying (BPSK) and QPSK, respectively. The results show that the BER performance of a DF relaying cooperative network using QPSK is within $2 \mathrm{~dB}$ of the ideal case of perfect impairment estimation when using the ML and SAGE estimator in combination with the proposed ML decoder. However, at high SNR the BER plot corresponding to the SAGE estimator deviates from that of the ML estimator due to the error floor of the proposed SAGE estimator as also depicted in Fig. 6.

Similar results are obtained for the case of AF relaying, where the gap between the cases of imperfect and perfect impairment estimation for BPSK and QPSK is in the range from 2 to $2.5 \mathrm{~dB}$ for both the proposed LS and SAGE estimators at low-to-mid SNRs ${ }^{9}$. This gap increases at high SNR for the proposed SAGE estimator due to the error floor of this estimator at high SNR as illustrated in Fig. 7. In Fig. 12(a), we also plot the BER results for a cooperative system that first employs the resynchronization filter in [15] to compensate MTOs and then attempts to remove MCFOs by employing the algorithm in [25]. This plot, which is denoted by " [15] and [24]" shows that such an approach fails to decode the received signal at $\mathbb{D}$ since the re-synchronization filter in [15] fails to compensate MTOs in the presence of MCFOs. Subsequently, the algorithm in [25] fails to nullify MCFOs, since the input signal is corrupted by MTOs. This corroborates our claim that previously proposed algorithms cannot decode the received signal in the presence of both MCFOs and MTOs. Finally, we note that in the case of BPSK the application of the proposed SAGE estimators and ML decoders results in an overall cooperative network BER of below $10^{-3}$ for SNRs greater than 16 and $24 \mathrm{~dB}$ for the DF and AF systems, respectively.

\section{CONCLUSION}

In this paper, training and data transmission methods for both DF-and AF-relaying multi-relay cooperative networks affected by MCFOs, MTOs, and unknown channel gains are presented. New closed-form FIM and CRLB expressions for the multiple parameter estimation problem are derived. The derived FIM shows that there exists coupling between the estimation errors of MCFOs, MTOs, and channel gains, which establishes that these parameters must be jointly estimated at the destination. In order to reduce overhead and complexity, two iterative estimators based on the ECM and SAGE algorithms are derived and their performance is compared against the CRLBs. Though global convergence of the proposed ECM and SAGE algorithm cannot be shown analytically, numerical simulations indicate that through proper initialization using an LS estimator the proposed estimators can obtain MCFOs, MTOs, and unknown channel gains jointly at the destination. In addition, it is established through computational complexity analyses that at SNR of $20 \mathrm{~dB}$ for a four-relay cooperative network, the proposed ECM and SAGE estimators are each over two orders of magnitude more computationally efficient than the previously proposed ML estimator in [17]. Next, an ML approach is proposed to decode the received signal at the destination for both DF and AF systems. Simulation results show that the combination of proposed estimators and ML decoder result in BER performance that is within $2-2.5 \mathrm{~dB}$ of that of a perfectly synchronized cooperative system. In spite of their advantages, the proposed estimators and ML decoder are sensitive to the initialization procedure and incur high computational complexity, respectively. Therefore, more research

\footnotetext{
${ }^{9}$ Similar results are obtained for the proposed ECM estimator.
} 
is needed to devise noniterative and computationally more efficient estimation and detection algorithms for achieving synchronization in cooperative networks.

\section{APPENDIX A}

DERIVATION OF $\mathbf{F}^{\mathrm{AF}}$

The $(\ell, q)$ th element of $4 K \times 4 K$ FIM is given by [31]

$$
\begin{aligned}
& {\left[\mathbf{F}^{[\mathrm{AF}]}(\overline{\boldsymbol{\theta}})\right]_{\ell, q}=2 \Re\left\{\frac{\partial \boldsymbol{\mu}_{\overline{\mathbf{y}}}^{H}}{\partial \bar{\theta}_{\ell}} \boldsymbol{\Sigma}_{\overline{\mathbf{y}}}^{-1} \frac{\partial \boldsymbol{\mu}_{\overline{\mathbf{y}}}}{\partial \bar{\theta}_{q}}\right\} } \\
&+\operatorname{Tr}\left(\boldsymbol{\Sigma}_{\overline{\mathbf{y}}}^{-1} \frac{\partial \boldsymbol{\Sigma}_{\overline{\mathbf{y}}}}{\partial \bar{\theta}_{\ell}} \boldsymbol{\Sigma}_{\overline{\mathbf{y}}}^{-1} \frac{\partial \boldsymbol{\Sigma}_{\overline{\mathbf{y}}}}{\partial \bar{\theta}_{q}}\right)
\end{aligned}
$$

where $\boldsymbol{\mu}_{\overline{\mathrm{y}}}=\overline{\boldsymbol{\Omega}} \boldsymbol{\alpha}$ and

$$
\begin{aligned}
{\left[\boldsymbol{\Sigma}_{\overline{\mathbf{y}}}\right]_{\ell, i}=} & \sum_{k=1}^{R} \sum_{m=1}^{R} \beta_{k} \beta_{m}^{*} \mathbb{E}\left[e^{j 2 \pi \ell \nu_{k}^{[r d]} / Q} \bar{t}_{k}^{[r]}\left(\ell T_{s}-\tau_{k}^{[r d]} T\right)\right. \\
& \left.\times\left(e^{j 2 \pi i \nu_{m}^{[r d]} / Q} \bar{t}_{m}^{r r]}\left(i T_{s}-\tau_{m}^{[r d]} T\right)\right)^{*}\right] \\
& \times \mathbb{E}\left[u_{k}(\ell) u_{m}^{*}(i)\right] \\
& +\mathbb{E}\left[w(\ell) w^{*}(i)\right], \\
= & \begin{cases}\sigma_{u}^{2} \sum_{k=1}^{K}\left|\beta_{k}\right|^{2}+\sigma_{w}^{2}, & i=\ell, k=m \\
0, & i \neq \ell, k \neq m\end{cases}
\end{aligned}
$$

where (A.2b) follows from (A.2a) due to the assumptions of unit amplitude PSK TSs and mutual independence of the noise at the relays and destination. Accordingly the derivatives in (A.1) can be derived as

$$
\begin{aligned}
\frac{\partial \boldsymbol{\mu}_{\overline{\mathbf{y}}}}{\partial \Re\left\{\alpha_{k}\right\}} & =-j \frac{\partial \boldsymbol{\mu}_{\overline{\mathbf{y}}}}{\partial \Im\left\{\alpha_{k}\right\}}=\left(\overline{\boldsymbol{\Lambda}}_{k} \overline{\mathbf{G}}_{k} \mathbf{t}_{s}\right) \odot \overline{\mathbf{t}}_{k}^{[r]}\left(\bar{\tau}_{k}\right), \\
\frac{\partial \boldsymbol{\mu}_{\overline{\mathbf{y}}}}{\partial \bar{\tau}_{k}} & =\left(\overline{\boldsymbol{\Lambda}}_{k} \overline{\mathbf{R}}_{k} \mathbf{t}_{s}\right) \odot \overline{\mathbf{t}}_{k}^{[r]}\left(\bar{\tau}_{k}\right) \alpha_{k}, \\
\frac{\partial \boldsymbol{\mu}_{\overline{\mathbf{y}}}}{\partial \bar{\nu}_{k}} & =j \mathbf{D}\left(\overline{\boldsymbol{\Lambda}}_{k} \overline{\mathbf{G}}_{k} \mathbf{t}_{s}\right) \odot \overline{\mathbf{t}}_{k}^{[r]}\left(\bar{\tau}_{k}\right) \alpha_{k}
\end{aligned}
$$

where $\overline{\mathbf{R}}_{k} \triangleq \partial \overline{\mathbf{G}}_{k} / \partial \bar{\tau}_{k}$ and $\partial \bar{t}_{k}^{[r]}(t) / \partial \bar{\tau}_{k}=0$. Since, $\boldsymbol{\Sigma}_{\overline{\mathbf{y}}}$ is not a function of MCFOs, MTOs, and channel gains $\boldsymbol{\alpha}$, we have

$$
\frac{\partial \boldsymbol{\Sigma}_{\overline{\mathbf{y}}}}{\partial \bar{\nu}_{k}}=\frac{\partial \boldsymbol{\Sigma}_{\overline{\mathbf{y}}}}{\partial \bar{\tau}_{k}}=\frac{\boldsymbol{\Sigma}_{\overline{\mathbf{y}}}}{\partial \Re\left\{\alpha_{k}\right\}}=\frac{\boldsymbol{\Sigma}_{\overline{\mathbf{y}}}}{\partial \Im\left\{\alpha_{k}\right\}}=0 .
$$

After substituting the derivatives in (A.3), (A.4), and (A.5) into (A.1) and carrying out straightforward algebraic manipulations, the upper triangular elements of FIM, for $\ell, q=1,2, \ldots, K$, can be obtained as

$$
\begin{aligned}
\mathbf{F}_{\ell, q}^{[\mathrm{AF}]} & \\
= & \left(2 / \sigma_{n}^{2}\right) \Re\left\{\left(\overline{\mathbf{t}}_{\ell}^{[r]}\left(\bar{\tau}_{l}\right)\right)^{H}\right. \\
& \left.\odot\left(\left(\mathbf{t}^{[s]}\right)^{H} \overline{\mathbf{G}}_{\ell}^{H} \overline{\boldsymbol{\Lambda}}_{\ell}^{H} \overline{\mathbf{\Lambda}}_{q} \overline{\mathbf{G}}_{q} \overline{\mathbf{t}}^{[s]}\right) \odot \overline{\mathbf{t}}_{q}^{[r]}\left(\bar{\tau}_{q}\right)\right\} \\
\mathbf{F}_{\ell, q+K}^{[\mathrm{AF}]} & \\
= & -\left(2 / \sigma_{n}^{2}\right) \Im\left\{\left(\overline{\mathbf{t}}_{\ell}^{[r]}\left(\bar{\tau}_{l}\right)\right)^{H}\right. \\
& \left.\odot\left(\left(\mathbf{t}^{[s]}\right)^{H} \overline{\mathbf{G}}_{\ell}^{H} \overline{\boldsymbol{\Lambda}}_{\ell}^{H} \overline{\boldsymbol{\Lambda}}_{q} \overline{\mathbf{G}}_{q} \mathbf{t}^{[s]}\right) \odot \overline{\mathbf{t}}_{q}^{[r]}\left(\bar{\tau}_{q}\right)\right\}
\end{aligned}
$$

$$
\begin{aligned}
& \mathbf{F}_{\ell+K, q+K}^{[\mathrm{AF}]} \\
& =\left(2 / \sigma_{n}^{2}\right) \Re\left\{\left(\overline{\mathbf{t}}_{\ell}^{[r]}\left(\bar{\tau}_{l}\right)\right)^{H}\right. \\
& \left.\odot\left(\left(\mathbf{t}^{[s]}\right)^{H} \overline{\mathbf{G}}_{\ell}^{H} \overline{\boldsymbol{\Lambda}}_{\ell}^{H} \overline{\boldsymbol{\Lambda}}_{q} \overline{\mathbf{G}}_{q} \mathbf{t}^{[s]}\right) \odot \overline{\mathbf{t}}_{q}^{[r]}\left(\bar{\tau}_{q}\right)\right\} \\
& \mathbf{F}_{\ell, q+2 K}^{[\mathrm{AF}]} \\
& =-\left(2 / \sigma_{n}^{2}\right) \Im\left\{\left(\overline{\mathbf{t}}_{\ell}^{[r]}\left(\bar{\tau}_{l}\right)\right)^{H}\right. \\
& \left.\odot\left(\left(\mathbf{t}^{[s]}\right)^{H} \overline{\mathbf{G}}_{\ell}^{H} \overline{\boldsymbol{\Lambda}}_{\ell}^{H} \mathbf{D} \overline{\boldsymbol{\Lambda}}_{q} \overline{\mathbf{G}}_{q} \mathbf{t}^{[s]}\right) \odot \overline{\mathbf{t}}_{q}^{[r]}\left(\bar{\tau}_{q}\right) \alpha_{q}\right\} \\
& \mathbf{F}_{\ell+K, q+2 K}^{[\mathrm{AF}]} \\
& =\left(2 / \sigma_{n}^{2}\right) \Re\left\{\left(\overline{\mathbf{t}}_{\ell}^{[r]}\left(\bar{\tau}_{l}\right)\right)^{H}\right. \\
& \left.\odot\left(\left(\mathbf{t}^{[s]}\right)^{H} \overline{\mathbf{G}}_{\ell}^{H} \overline{\boldsymbol{\Lambda}}_{\ell}^{H} \mathbf{D} \overline{\mathbf{\Lambda}}_{q} \overline{\mathbf{G}}_{q} \mathbf{t}^{[s]}\right) \odot \overline{\mathbf{t}}_{q}^{[r]}\left(\bar{\tau}_{q}\right) \alpha_{q}\right\} \\
& \mathbf{F}_{\ell+2 K, q+2 K}^{[\mathrm{AF}]} \\
& =\left(2 / \sigma_{n}^{2}\right) \Re\left\{\alpha_{\ell}^{*}\left(\overline{\mathbf{t}}_{\ell}^{[r]}\left(\bar{\tau}_{l}\right)\right)^{H}\right. \\
& \left.\odot\left(\left(\mathbf{t}^{[s]}\right)^{H} \overline{\mathbf{G}}_{\ell}^{H} \overline{\boldsymbol{\Lambda}}_{\ell}^{H} \mathbf{D}^{2} \overline{\boldsymbol{\Lambda}}_{q} \overline{\mathbf{G}}_{q} \mathbf{t}^{[s]}\right) \odot \overline{\mathbf{t}}_{q}^{[r]}\left(\bar{\tau}_{q}\right) \alpha_{q}\right\} \\
& \mathbf{F}_{\ell, q+3 K}^{[\mathrm{AF}]} \\
& =\left(2 / \sigma_{n}^{2}\right) \Re\left\{\left(\overline{\mathbf{t}}_{\ell}^{[r]}\left(\bar{\tau}_{l}\right)\right)^{H}\right. \\
& \left.\odot\left(\left(\mathbf{t}^{[s]}\right)^{H} \overline{\mathbf{G}}_{\ell}^{H} \overline{\boldsymbol{\Lambda}}_{\ell}^{H} \overline{\boldsymbol{\Lambda}}_{q} \overline{\mathbf{R}}_{q} \mathbf{t}^{[s]}\right) \odot \overline{\mathbf{t}}_{q}^{[r]}\left(\bar{\tau}_{q}\right) \alpha_{q}\right\} \\
& \mathbf{F}_{\ell+K, q+3 K}^{[\mathrm{AF}]} \\
& =\left(2 / \sigma_{n}^{2}\right) \Im\left\{\left(\overline{\mathbf{t}}_{\ell}^{[r]}\left(\bar{\tau}_{l}\right)\right)^{H}\right. \\
& \left.\odot\left(\left(\mathbf{t}^{[s]}\right)^{H} \overline{\mathbf{G}}_{\ell}^{H} \overline{\boldsymbol{\Lambda}}_{\ell}^{H} \overline{\boldsymbol{\Lambda}}_{q} \overline{\mathbf{R}}_{q} \mathbf{t}^{[s]}\right) \odot \overline{\mathbf{t}}_{q}^{[r]}\left(\bar{\tau}_{q}\right) \alpha_{q}\right\} \\
& \mathbf{F}_{\ell+2 K, q+3 K}^{[\mathrm{AF}]} \\
& =\left(2 / \sigma_{n}^{2}\right) \Im\left\{\alpha_{\ell}^{*}\left(\overline{\mathbf{t}}_{\ell}^{[r]}\left(\bar{\tau}_{l}\right)\right)^{H}\right. \\
& \left.\odot\left(\left(\mathbf{t}^{[s]}\right)^{H} \overline{\mathbf{G}}_{\ell}^{H} \overline{\boldsymbol{\Lambda}}_{\ell}^{H} \mathbf{D} \overline{\mathbf{\Lambda}}_{q} \overline{\mathbf{R}}_{q} \mathbf{t}^{[s]}\right) \odot \overline{\mathbf{t}}_{q}^{[r]}\left(\bar{\tau}_{q}\right) \alpha_{q}\right\} \\
& \mathbf{F}_{\ell+3 K, q+3 K}^{[\mathrm{AF}]} \\
& =\left(2 / \sigma_{n}^{2}\right) \Re\left\{\alpha_{\ell}^{*}\left(\overline{\mathbf{t}}_{\ell}^{[r]}\left(\bar{\tau}_{l}\right)\right)^{H}\right. \\
& \left.\odot\left(\left(\mathbf{t}^{[s]}\right)^{H} \overline{\mathbf{R}}_{\ell}^{H} \overline{\boldsymbol{\Lambda}}_{\ell}^{H} \overline{\boldsymbol{\Lambda}}_{q} \overline{\mathbf{R}}_{q} \mathbf{t}^{[s]}\right) \odot \overline{\mathbf{t}}_{q}^{[r]}\left(\bar{\tau}_{q}\right) \alpha_{q}\right\} .
\end{aligned}
$$

Note that the lower triangular elements of $\mathbf{F}^{[\mathrm{AF}]}$ can be easily obtained by simple manipulation of (A.6)-(A.15) and are not included here for brevity. 


\section{REFERENCES}

[1] A. Sendonaris, E. Erkip, and B. Aazhang, "User cooperative diversity_Part I: System description; part II: Implementation and performance analysis," IEEE Trans. Commun., vol. 51, no. 11, pp. 1927-1948, Nov. 2003.

[2] J. Yindi and H. Jafarkhani, "Network beamforming using relays with perfect channel information," IEEE Trans. Acoust., Speech, Signal Processing, vol. 3, no. 6, pp. 473-476, Apr. 2007.

[3] Y. Zheng, H. Mehrpouyan, and S. D. Blostein, "Application of phase shift in coherent multi-relay MIMO communications," in Proc IEEE Int. Conf. Commun. (ICC), Dresden, Germany, 2009.

[4] J. N. Laneman and G. W. Wornell, "Distributed space-time-coded protocols for exploiting cooperative diversity in wireless networks," IEEE Trans. Inf. Theory, vol. 49, no. 10, pp. 2415-2425, Oct. 2003.

[5] J. N. Laneman, D. N. C. Tse, and G. W. Wornell, "Cooperative diversity in wireless networks: Efficient protocols and outage behavior," IEEE Trans. Inf. Theory, vol. 50, no. 12, pp. 3062-3080, Dec. 2004.

[6] X. Li, C. Xing, Y.-C. Wu, and S. C. Chan, "Timing estimation and resynchronization for amplify-and-forward communication systems," IEEE Trans. Signal Processing, vol. 58, no. 4, pp. 2218-2229, Apr. 2010.

[7] H. Mehrpouyan and S. D. Blostein, "Estimation, training, and effect of timing offsets in distributed cooperative networks," in Proc. IEEE Global Commun. Conf. (GLOBECOM), Miami, FL, Dec. 2010.

[8] H. Mehrpouyan and S. D. Blostein, "Bounds and algorithms for multiple frequency offset estimation in cooperative networks," IEEE Trans. Wireless Commun., vol. 10, no. 4, pp. 1300-1311, Apr. 2011.

[9] O. Besson and P. Stoica, "On parameter estimation of MIMO flat-fading channels with frequency offsets," IEEE Trans. Signal Processing, vol. 51, no. 3, pp. 602-613, Mar. 2003.

[10] T. Pham, A. Nallanathan, and Y. Liang, "Joint channel and frequency offset estimation in distributed MIMO flat-fading channels," IEEE Trans. Wireless Commun., vol. 7, no. 2, pp. 648-656, Feb. 2008.

[11] Y. Yao and T. Ng, "Correlation-based frequency offset estimation in MIMO system," in Proc. IEEE Veh. Technol. Conf. (VTC), Orlando, FL, Oct. 2003.

[12] Z. Lu, J. Li, L. Zhao, and J. Pang, "Iterative parameter estimation in MIMO flat-fading channels with frequency offsets," in Proc IEEE Int. Conf. Advanced Inf. Netw. Appl. (AINA), Austria, Apr. 2006.

[13] Q. Jiang, K. Zhang, J. Liu, and G. Shen, "Joint carrier frequency offset and channel estimation for AF cooperative OFDM systems," Springer J. Wireless Pers. Commun., vol. 55, pp. 1-27, Oct. 2010.

[14] M. T. Hossain, D. B. Smith, and S. Kandeepan, "Timing synchronization for cooperative communications with detect and forward relaying," Springer J. Wireless Pers. Commun., 2010.

[15] X. Li, Y. C. Wu, and E. Serpedin, "Timing synchronization in decode-and-forward cooperative communication systems.," IEEE Trans. Signal Processing, vol. 57, no. 4, pp. 1444-1455, Apr. 2009.

[16] F. Gao, T. Cui, and A. Nallanathan, "On channel estimation and optimal training design for amplify-and-forward relay networks," IEEE Trans. Wireless Commun., vol. 7, no. 5, pp. 1907-1916, May 2008.

[17] Y. Tian, X. Lei, Y. Xiao, and S. Li, "ML synchronization algorithm and estimation bounds for cooperative systems," in Proc IEEE Pacific Asia Conf. on Circuits, Commun. and Systems (PACCS), China, Aug. 2010.

[18] Y. X. S. L. Y. Tian and X. Lei, "SAGE based joint timing-frequency offsets and channel estimation in distributed MIMO systems," Elsevier J. Comput. Commun., vol. 33, no. 17, pp. 2125-2131, July 2010.

[19] L. Dai, Z. Wang, J. Wang, and Z. Yang, "Joint channel estimation and time-frequency synchronization for uplink TDS-OFDMA systems," IEEE Trans. Consumer Electron., vol. 56, no. 2, pp. 494-500, May 2010.

[20] J.-H. Lee and S.-C. Kim, "Time and frequency synchronization for OFDMA uplink system using the SAGE algorithm," IEEE Trans. Wireless Commun., vol. 6, no. 4, pp. 1176-1181, Apr. 2007.

[21] M. Morelli, "Time and frequency synchronization for the uplink of an OFDMA system,” IEEE Trans. Commun., vol. 52, no. 2, pp. 296-396, Feb. 2004.

[22] M.-O. Pun, M. Morelli, and C.-C. J. Kuo, "Maximum likelihood synchronization and channel estimation for OFDMA uplink transmissions," IEEE Trans. Commun., vol. 54, no. 4, pp. 726-736, Apr. 2006.

[23] H. Wang, X.-G. Xia, and Q. Yin, "Computationally efficient equalization for asynchronous cooperative communications with multiple frequency offsets," IEEE Trans. Wireless Commun., vol. 8, no. 2, pp. 648-655, Feb. 2009.
[24] F. Tian, X.-G. Xia, and P. C. Ching, "Signal detection in a cooperative communication system with multiple CFOs by exploiting the properties of space-frequency codes," in Proc. IEEE Int. Conf. Commun. (ICC), Beijing, China, Jun. 2008.

[25] D. Veronesi and D. L. Goeckel, "Multiple frequency offset compensation in cooperative wireless systems," in Proc. IEEE Global Commun. Conf. (GLOBECOM), San Francisco, CA, Dec. 2006.

[26] A. Kannan, T. P. Krauss, and M. D. Zoltowski, "Separation of cochannel signals under imperfect timing and carrier synchronization," IEEE Trans. Veh. Technol., vol. 50, no. 1, pp. 79-96, Jan. 2001.

[27] H. Meyr, M. Moeneclaey, and S. A. Fechtel, Digital Communication Receivers, Synchronization, Channel Estimation, and Signal Processing, ser. Telecom. and Signal Processing, J. G. Proakis, Ed. Hoboken, NJ: Wiley, 1998.

[28] H. Mehrpouyan and S. D. Blostein, "Comments on "timing estimation and resynchronization for amplify-and-forward communication systems"," IEEE Signal Processing Lett., vol. 59, pp. 4047-4048, Aug. 2011.

[29] X. Li, C. Xing, Y. C. Wu, and S. C. Chan, "Authors reply to comments on timing estimation and resynchronization for amplify-and-forward communication systems," IEEE Signal Processing Lett., vol. 59, pp. 4048-4049, Aug. 2011.

[30] R. A. Horn and C. R. Johnson, Matrix Analysis. Cambridge, U.K.: Cambridge University Press, 1990.

[31] S. M. Kay, Fundamentals of Statistical Signal Processing: Estimation Theory. Englewood Cliffs, NJ: Prentice Hall, 1993.

[32] I. Ziskand and M. Wax, "Maximum likelihood localization of multiple sources by Alternating projection," IEEE Trans. Acoust., Speech, Signal Processing, vol. 36, no. 10, pp. 1553-1560, Oct. 1988.

[33] T. K. Moon, "The expectation-maximazation algorithm," IEEE Signal Processing Mag., vol. 13, pp. 47-60, Nov. 1996.

[34] M. Feder and E. Weinstein, "Parameter estimation of superimposed signals using the EM algorithm," IEEE Trans. Acoust., Speech, Signal Processing, vol. 36, no. 4, pp. 477-489, Apr. 1988.

[35] G. J. McLachlan and T. Krishnan, The EM Algorithm and Extensions. Hoboken, NJ: Wiley, 2008.

[36] X. L. Meng and D. B. Rubin, "Maximum likelihood estimation via the ECM algorithm: A general framework," Biometrika, vol. 8, no. 2, pp. 267-278, Jun. 1993.

[37] R. J. Leveque, Finite Difference Methods for Ordinary and Partial Differntial Equations. Philadelphia, PA: Society for Industrial and Applied Mathematics (SIAM), 2007.

[38] T. A. Fesler and A. O. Hero, "Space-alternating generalized expectation maximization algorithm," IEEE Trans. Signal Processing, vol. 42, no. 10, pp. 2664-2677, Oct. 1994.

[39] N. Moller, "On Schoonhage's algorithm and subquadratic integer gcd computation," Math. Comput., vol. 77, pp. 589-607, Jan. 2008.

[40] B. P. Singh and R. Singh, Electronic Devices and Integrated Circuits. Delhi, India: Pearson Education India, 2006.

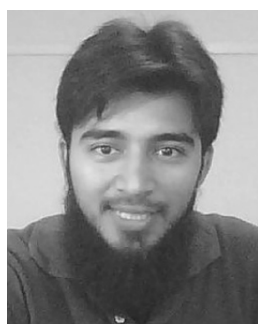

Ali A. Nasir (S'11) received the B.Sc. degree (with first-class honors) in electrical engineering from the University of Engineering and Technology (UET), Lahore, Pakistan, in 2007. He is currently pursuing the Ph.D. degree from the Research School of Engineering, the Australian National University (ANU), Canberra, Australia.

Prior to joining ANU, he worked as a Lecturer in UET for nine months and then as a Design Engineer in the Centre for Advanced Research in Engineering (CARE), Islamabad, Pakistan for a year. He was a Research Visitor in Chalmers University of Technology, Gothenburg, Sweden, from April to October 2011. His research interests are in the area of synchronization and channel estimation in cooperative communication, MIMO and OFDM systems.

Mr. Nasir was awarded a University Gold Medal for outstanding performance during the final year of his undergraduate studies. He is a recipient of an ANU International Ph.D. scholarship for the duration of his doctorate. He was also awarded an ANU Vice Chancellor's Higher Degree Research (HDR) travel grant in 2011. 


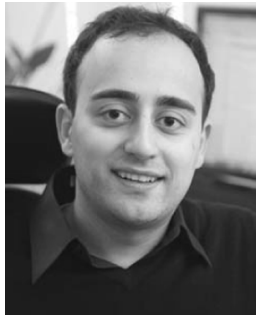

Hani Mehrpouyan (S'05-M'10) received the B.Sc. degree (with honors) in computer engineering from Simon Fraser University, Burnaby, Canada, in 2004. In 2005, he joined the Department of Electrical and Computer Engineering at Queen's University, Kingston, ON, Canada, as a doctoral candidate, where he received the Ph.D. degree in electrical engineering in 2010.

Since September of 2010, he has been with the Department of Signals and Systems at Chalmers University of Technology, Sweden, as a Postdoctoral Researcher. His current research interests are in the areas of applied signal processing and physical layers of wireless communication systems, including synchronization, channel estimation, and performance optimization. He has also been involved with industry leaders such as Ericsson AB, Research in Motion (RIM), and Alcatel and has more than 20 publications in IEEE journals and conferences.

Dr. Mehrpouyan has received more than 10 scholarships and awards due to leadership, academic, research, and teaching excellence, e.g., IEEE Globecom Early Bird Student Award, NSERC-IRDF, NSERC PGS-D, NSERC CGS-M Alexander Graham Bell, B.C. Wireless Innovation, and more.

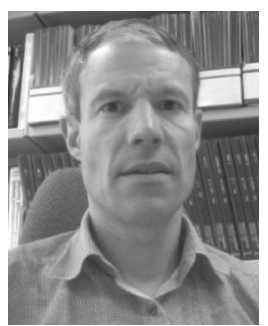

Steven D. Blostein (S'83-M'88-SM'96) received the B.S. degree in electrical engineering from Cornell University, Ithaca, NY, in 1983, and the M.S. and $\mathrm{Ph} . \mathrm{D}$. degrees in electrical and computer engineering from the University of Illinois, Urbana-Champaign, in 1985 and 1988, respectively.

He has been on the faculty in the Department of Electrical and Computer Engineering Queen's University, Kingston, ON, Canada, since 1988 and currently holds the position of Professor. From 2004 to 2009, he was Department Head. From 1999 to 2003 , he was the leader of the Multi-Rate Wireless Data Access Major Project sponsored by the Canadian Institute for Telecommunications Research. He has also been a consultant to industry and government in the areas of image compression, target tracking, radar imaging, and wireless communications. He spent sabbatical leaves at Lockheed Martin Electronic Systems and at Communications Research Centre in Ottawa, Canada. His current interests lie in the application of signal processing to problems in wireless communications systems, including synchronization, network MIMO, and physical layer optimization for multimedia transmission.

Dr. Blostein has been a member of the Samsung 4G Wireless Forum as well as an invited distinguished speaker. He served as Chair of IEEE Kingston Section (1994), Chair of the Biennial Symposium on Communications (2000, 2006, and 2008), Associate Editor for IEEE TRANSACTIONS ON IMAGE Processing (1996-2000), and Publications Chair for IEEE ICASSP 2004. For a number of years, he has been serving on technical program committees for IEEE Communications Society conferences that include ICC, Globecom, and WCNC. He has been serving as an Editor of IEEE TRANSACTIONS ON Wireless COMMUNICATIONS since 2007. He is a registered Professional Engineer in Ontario.

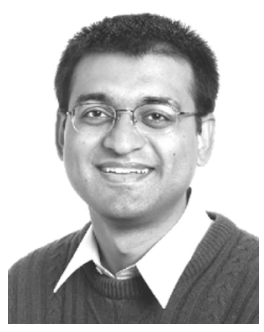

Salman Durrani (S'00-M'05-SM'10) received the B.Sc. degree (with first class honors) in electrical engineering from the University of Engineering and Technology, Lahore, Pakistan, in 2000. He received the Ph.D. degree in electrical engineering from the University of Queensland, Brisbane, Australia, in December 2004

Since March 2005, he has been a Lecturer in the Research School of Engineering, College of Engineering and Computer Science, The Australian National University, Canberra, Australia. His current research interests are in wireless communications and signal processing, including synchronization in cooperative communication systems, connectivity of ad-hoc networks, and vehicular networks and signal processing on the unit sphere.

Dr. Durrani serves as a Technical Program Committee Member for international conferences such as ICC'12, PIMRC'11 and AusCTW'12. He was a recipient of an International Postgraduate Research Scholarship from the Australian Commonwealth during 2001-2004. He was awarded a University Gold Medal during his undergraduate studies. He has 47 publications to date in refereed international journals and conferences. He is a Member of Institution of Engineers, Australia.

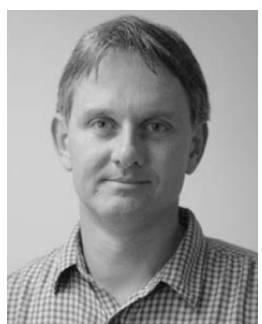

Rodney A. Kennedy (S'86-M'88-SM'01-F'05) received the B.E. degree from the University of New South Wales, Sydney, Australia, the M.E. degree from the University of Newcastle, and the Ph.D degree from the Australian National University, Canberra.

$\mathrm{He}$ is currently a Professor in the Research School of Engineering, Australian National University. His research interests include digital signal processing, digital and wireless communications, and acoustical signal processing. 